JOURNAL OF

SYMPLECTIC GEOMETRY

Volume 1 Number 4, 785-828, 2002

\title{
CONTACT TORIC MANIFOLDS
}

\section{EUGENE LERMAN}

We provide a complete and self-contained classification of (compact connected) contact toric manifolds thereby finishing the work initiated by Banyaga and Molino and by Galicki and Boyer. Our motivation comes from the conjectures of Toth and Zelditch on the uniqueness of toric integrable actions on the punctured cotangent bundles of the $n$-torus $\mathbb{T}^{n}$ and of the two-sphere $S^{2}$. The conjectures are equivalent to the uniqueness, up to conjugation, of maximal tori in the contactomorphism groups of the cosphere bundles of $\mathbb{T}^{n}$ and $S^{2}$ respectively.

\section{Contents}

1. Introduction

2. Group actions on contact manifolds

3. Local structure of contact toric manifolds

4. Properties of contact moment maps

5. From local to global

6. Proof of the classification theorem

6.1. Proof of Theorem 2.18 (1)

6.2. Proof of Theorem 2.18 (2)

6.3. Proof of Theorem 2.18 (3)

6.4. Proof of Theorem 2.18 (4)

7. Uniqueness of toric integrable actions on the punctured cotangent bundles of $\mathbb{T}^{n}$ and $S^{2}$ 


\section{Introduction}

In a recent paper Toth and Zelditch studied the relation between the dynamics of the geodesic flow on a compact Riemannian manifold $(Q, g)$ and the growth rate of $L^{\infty}$ norms of $L^{2}$-normalized eigenfunctions of the Laplace operator $\Delta_{g}[\mathbf{T Z}]$. They showed that if the square root of the Laplace operator $\sqrt{\Delta_{g}}$ is "quantum completely integrable" and has uniformly bounded eigenfunctions then the metric $g$ is flat, and hence by the Bieberbach theorems $Q$ is finitely covered by a torus. The proof is particularly transparent when the geodesic flow is toric integrable. The latter means that there is an effective action of a torus $\mathbb{T}^{n}, n=\operatorname{dim} Q$, on the punctured cotangent bundle $T^{*} Q \backslash Q$ of $Q$ which

1) commutes with dilations $\rho_{t}: T^{*} Q \backslash Q \rightarrow T^{*} Q \backslash Q, \rho(q, p)=$ $\left(q, e^{t} p\right)$

2) preserves the standard symplectic form on $T^{*} Q$ and

3) preserves the energy function $h(q, p)=g_{q}^{*}(p, p)$, where $g^{*}$ denotes the metric on $T^{*} Q$ dual to $g$. (The Hamiltonian flow of $h$ is the geodesic flow.)

Note that any symplectic group action on the punctured cotangent bundle which commutes with dilations preserves the Liouville 1-form and is, therefore, Hamiltonian. Consequently if a metric on a manifold $Q$ is toric integrable, the pull-back metric on a finite cover of $Q$ is toric integrable as well. One is therefore lead to wonder if in the case where $Q$ is a torus, the boundedness of eigenfunctions is necessary for the flatness of the metric or if toric integrability by itself is enough. The latter is indeed the case, as N. Shirokova and I proved in $[\mathbf{L S}]$ :

Theorem 1. Suppose that $g$ is a toric integrable metric on a torus $\mathbb{T}^{n}:=\mathbb{R}^{n} / \mathbb{Z}^{n}$. Then $g$ is flat.

Toth and Zelditch showed using hard results of Mañe and of Burago and Ivanov that the proof of Theorem 1 can be reduced to the following proposition.

Proposition 1.1. Any (effective) action of the $n$ torus $\mathbb{T}^{n}$ on the punctured cotangent bundle $T^{*} \mathbb{T}^{n} \backslash \mathbb{T}^{n}$, which preserves the symplectic form and commutes with dilations, is free.

We will refer to the torus actions satisfying the hypotheses of Proposition 1.1 as toric integrable. 
Toth and Zelditch additionally conjectured that there is only one such action of $\mathbb{T}^{n}$ (up to an isomorphism): the lift of the left multiplication. Any action of a group $G$ on a punctured cotangent bundle $T^{*} Q \backslash Q$ which commutes with dilations induces an action of $G$ on the orbit space $S\left(T^{*} Q\right):=\left(T^{*} Q \backslash Q\right) / \mathbb{R}$, which is the cosphere bundle of $Q$. Moreover any such action which is symplectic gives rise to a contact action on $S\left(T^{*} Q\right)$ (for more on contact actions see section 2 below). Therefore Proposition 1.1 and hence Theorem 1 are a consequence of the proposition below:

Proposition 1.2. Any (effective) contact action of $\mathbb{T}^{n}$ on the cosphere bundle $S\left(T^{*} \mathbb{T}^{n}\right)$ is free.

A proof of Proposition 1.2 is the content of $[\mathbf{L S}]$. Note that $\operatorname{dim} S\left(T^{*} \mathbb{T}^{n}\right)$ $=2 \operatorname{dim} \mathbb{T}^{n}-1$. It is therefore reasonable to define a contact toric manifold to be a triple of the form $(M, \xi, \tau)$ where $M$ is a manifold of dimension $2 n-1, \xi$ a contact structure on $M$ and $\tau: \mathbb{T}^{n} \times M \rightarrow M$ is an effective action of a torus $\mathbb{T}^{n}$ preserving $\xi$.

When Shirokova and I started working on the questions of Toth and Zelditch we did a literature search and were happy to discover the work of Banyaga and Molino [BaM1]. (Professor Banyaga kindly provided us with a copy of [BaM2] which is, as far as I can tell, an updated version of [BaM1].) Unfortunately neither the results in [BaM2] nor the later work of Boyer and Galicki $[\mathrm{BG}]$ were strong enough for our purposes. Shirokova and I ended up giving a topological classification of compact connected toric manifolds with non-free torus actions and proving that no such manifold could have the homotopy type of $S\left(T^{*} \mathbb{T}^{n}\right) \simeq \mathbb{T}^{n} \times S^{n-1}$. An interesting question, however, remained open - what are all (compact connected) contact toric manifolds? An answer to this question is the content of the present paper. It turns out that

Compact connected contact toric manifolds are classified by rational polyhedral cones on simple polytopes with exceptional series occurring in dimensions 3 and 5 .

A precise statement of the classification is given in Theorem 2.18 below. The classification allows us to strengthen Propositions 1.2, thereby proving a conjecture posed in in $[\mathbf{T Z}]$ :

Theorem 1.3. Up to appropriate isomorphisms there is only one effective contact action the n-torus $\mathbb{T}^{n}$ on the co-sphere bundle $S\left(T^{*} \mathbb{T}^{n}\right)$.

REMARK 1.4. Theorem 1.3 is equivalent to 
1) There is only one toric integrable action of $\mathbb{T}^{n}$ on the punctured cotangent bundle $T^{*} \mathbb{T}^{n} \backslash \mathbb{T}^{n}$.

2) Up to conjugation there is only one copy of the torus $\mathbb{T}^{n}$ in the group of the contactomorphisms $\operatorname{Diff}\left(S\left(T^{*} \mathbb{T}^{n}\right), \xi\right.$ ) (here $\xi$ denotes the standard contact structure on $S\left(T^{*} \mathbb{T}^{n}\right)$ ). That is, maximal tori in $\operatorname{Diff}\left(S\left(T^{*} \mathbb{T}^{n}\right), \xi\right)$ are unique up to conjugation.

Similarly we will prove:

Theorem 1.5. Up to appropriate isomorphisms there is only one effective contact action of $\mathbb{T}^{2}$ on $S\left(T^{*} S^{2}\right)$.

REMARK 1.6. Theorem 1.5 is equivalent to

1) There is only one toric integrable action of $\mathbb{T}^{2}$ on the punctured cotangent bundle $T^{*} S^{2} \backslash S^{2}$.

2) The maximal tori in the group of contactomorphisms $\operatorname{Diff}\left(S\left(T^{*} S^{2}\right), \xi\right)$ are unique up to conjugation.

Note, by way of contrast, that for every integer $k>1$ there is a contact structure $\xi_{k}$ on the cosphere bundle of $S^{3}$ such that the group of contactomorphisms $\operatorname{Diff}\left(S\left(T^{*} S^{3}\right), \xi_{k}\right)$ has at least $k$ non-conjugate maximal tori $[\mathbf{L} 3]$.

I suspect that there are preciously few manifolds that admit toric integrable geodesic flows. In fact the only examples I know are tori and the low-dimensional spheres, and it would be surprising if there are others. Note that it is an entirely different problem to classify all toric integrable metrics on a given manifold. As was mentioned earlier, any toric integrable metric on a torus has to be flat and conversely, any flat metric is toric integrable. But even for the 2-sphere the classification of toric integrable metrics s open.

We conclude the introduction by saying a few words about the organization of the paper. In section 2 we recall some basic notions of contact geometry, discuss contact group actions and corresponding moment maps, recall the notion of a contact quotient, define contact toric manifolds and their isomorphisms and set up notation in order to state the classification theorem, Theorem 2.18. In section 3 we define pre-isotropic embeddings (by analogy with pre-Lagrangian embeddings), prove an equivariant version of the tubular neighborhood for pre-isotropic embeddings and write out the local normal form theorem for a contact moment map in a neighborhood of a pre-isotropic orbit. The local normal form theorem is then applied to contact toric manifolds. Many of the results of this section are not original and 
have appeared elsewhere, such as in [BaM1, BaM2], in a somewhat different form. In section 4 we gather a number of results describing the properties of contact moment maps, such as convexity of the image and connectedness of the fibers, and then apply them to moment maps on contact toric manifolds. It is interesting to note that convexity and connectedness fail in dimension 3 . In section 5 we prove that compact connected contact toric manifolds are classified by the elements of the first Čech cohomology of their orbit spaces with coefficients in a certain sheaf. In section 6 we put together the results of sections 4 and 5 to prove the classification theorem. Section 7 contains two applications of the classification theorem: we prove Theorems 1.3 and 1.5 on the uniqueness of toric integrable actions on the punctured cotangent bundles $T^{*} \mathbb{T}^{N} \backslash \mathbb{T}^{n}$ and $T^{*} S^{2} \backslash S^{2}$.

A note on notation. If $U$ is a subspace of a vector space $V$ we denote its annihilator in the dual vector space $V^{*}$ by $U^{\circ}$. Thus $U^{\circ}=\left\{\ell \in V^{*} \mid\right.$ $\left.\left.\ell\right|_{U}=0\right\}$.

Throughout the paper the Lie algebra of a Lie group denoted by a capital Roman letter is denoted by the same small letter in the fraktur font: thus $\mathfrak{g}$ denotes the Lie algebra of a Lie group $G$ etc. The vector space dual to $\mathfrak{g}$ is denoted by $\mathfrak{g}^{*}$. The identity element of a Lie group is denoted by 1 . The natural pairing between $\mathfrak{g}$ and $\mathfrak{g}^{*}$ is denoted by $\langle\cdot, \cdot\rangle$.

When a Lie group $G$ acts on a manifold $M$ we denote the action by an element $g \in G$ on a point $x \in M$ by $g \cdot x ; G \cdot x$ denotes the $G$-orbit of $x$ and so on. The vector field induced on $M$ by an element $X$ of the Lie algebra $\mathfrak{g}$ of $G$ is denoted by $X_{M}\left(\right.$ that is, $\left.X_{M}(x)=\left.\frac{d}{d t}\right|_{0}(\exp t X) \cdot x\right)$ and the diffeomorphism induced by $g \in G$ on $M$ by $g_{M}$. Thus in this notation $g \cdot x=g_{M}(x)$. The isotropy group of a point $x \in M$ is denoted by $G_{x}$; the Lie algebra of $G_{x}$ is denoted by $\mathfrak{g}_{x}$ and is referred to as the isotropy Lie algebra of $x$. Recall that $\mathfrak{g}_{x}=\left\{X \in \mathfrak{g} \mid X_{M}(x)=0\right\}$.

If a Lie group $G$ is a torus we denote the integral lattice of $G$ by $\mathbb{Z}_{G}$ and the dual weight lattice by $\mathbb{Z}_{G}^{*}$. Recall that $\mathbb{Z}_{G}=\operatorname{ker}(\exp : \mathfrak{g} \rightarrow G)$, $\mathbb{Z}_{G}^{*}=\operatorname{Hom}_{\mathbb{Z}}\left(\mathbb{Z}_{G}, \mathbb{Z}\right)$.

If $X$ is a vector field and $\tau$ is a tensor, then $L_{X} \tau$ denotes the Lie derivative of $\tau$ with respect to $X$.

If $P$ is a principal $G$-bundle then $[p, m]$ denotes the point in the associated bundle $P \times{ }_{G} M=(P \times M) / G$ which is the orbit of $(p, m) \in$ $P \times M$.

The symbol $\alpha$ always denotes a contact form and $\xi$ always denotes a co-oriented contact structure. 
Acknowledgments. This work was written during a visit of the American Institute of Mathematics and of the University of California at Berkeley.

\section{Group actions on contact manifolds}

In this section we recall the basic notions of contact geometry, discuss contact group actions and corresponding moment maps, recall the notion of a contact quotient, define contact toric manifolds and their isomorphisms and set up the notation in order to state the main classification theorem, Theorem 2.18.

Definition 2.1. Recall that a 1-form $\alpha$ on a manifold $M$ is contact if $\alpha_{x} \neq 0$ for any $x \in M$, so $\xi=\operatorname{ker} \alpha$ is a codimension- 1 distribution, and if additionally $\left.d \alpha\right|_{\xi}$ is non-degenerate. Thus the vector bundle $\xi \rightarrow M$ necessarily has even-dimensional fibers, and the manifold $M$ is necessarily odd-dimensional.

Definition 2.2. A codimension-1 distribution $\zeta$ on a manifold $M$ is co-orientable if its annihilator $\zeta^{\circ} \subset T^{*} M$ is an oriented line bundle, i.e., has a nowhere vanishing global section. It is co-oriented if one component $\zeta_{+}^{\circ}$ of $\zeta^{\circ} \backslash 0$ ( $\zeta^{\circ}$ minus the zero section $)$ is chosen.

Definition 2.3. A co-oriented contact structure $\xi$ on a manifold $M$ is a co-oriented codimension-1 distribution such that $\xi^{\circ} \backslash 0$ is a symplectic submanifold of the cotangent bundle $T^{*} M$ (the cotangent bundle is given the canonical symplectic form). We denote the chosen component of $\xi^{\circ} \backslash 0$ by $\xi_{+}^{\circ}$ and refer to it as the symplectization of $(M, \xi)$.

REMARK 2.4. It is a standard fact that $\xi \subset T M$ is a co-oriented contact structure if and only if there is a contact form $\alpha$ with $\operatorname{ker} \alpha=\xi$ (given $\xi$ choose $\alpha$ to be a section of $\left.\xi^{\circ} \backslash 0 \rightarrow M\right)$. If $f$ is any function on $M$ then $e^{f} \alpha$ defines the same contact structure $\xi$ and conversely, if $\alpha$ and $\alpha^{\prime}$ are two contact forms defining the same contact structure with the same co-orientation, then $\alpha^{\prime}=e^{f} \alpha$ for some function $f$. That is, a co-oriented contact structure is the same thing as a conformal class of contact forms.

REMARK 2.5. In this paper $\alpha$ always denotes a contact form and $\xi$ always denotes a co-oriented contact structure (with a co-orientation understood). We will refer to a pair $(M, \alpha)$ or to a pair $(M, \xi)$ as a (co-oriented) contact manifold. 
Lemma 2.6. Suppose a Lie group $G$ acts properly on a manifold $M$ preserving a co-oriented codimension-1 distribution $\zeta$ and its coorientation. That is, suppose the lifted action of $G$ on $T^{*} M$ preserves a component $\zeta_{+}^{\circ}$ of $\zeta^{\circ} \backslash 0$. Then there is a $G$-invariant 1-form $\beta$ on $M$ such that $\zeta=\operatorname{ker} \beta$ and $\beta(M) \subset \zeta_{+}^{\circ}$.

Proof. To choose $\beta$ first choose any 1-form $\tilde{\beta}$ on $M$ with $\operatorname{ker} \tilde{\beta}=\zeta$ and $\tilde{\beta}(M) \subset \zeta_{+}^{\circ}$. If $G$ is compact, we then let $\beta$ to be the average $\int_{G}\left(g_{M}\right)^{*} \tilde{\beta} d g$ of $\tilde{\beta}$ over the group $G$. If $G$ is not compact, then due to the existence of slices we may assume that $M=G \times_{K} V$ for some representation $K \rightarrow G L(V)$ of a compact Lie group $K$. We average the restriction $\left.\tilde{\beta}\right|_{V}: V \rightarrow T^{*}\left(G \times_{K} V\right)$ over $K$ and then extend it to all of $M$ by $G$-invariance.

Therefore given a proper action of a Lie group $G$ on a contact manifold $(M, \xi=\operatorname{ker} \alpha)$ which preserves the co-orientation, we may (and do) assume that the contact form $\alpha$ is $G$-invariant.

Definition 2.7. If a Lie group $G$ acts on a manifold $M$ preserving a 1form $\beta$, the corresponding $\beta$-moment map $\Psi_{\beta}: M \rightarrow \mathfrak{g}^{*}$ determined by $\beta$ is defined by

$$
\left\langle\Psi_{\beta}(x), X\right\rangle=\beta_{x}\left(X_{M}(x)\right)
$$

for all $x \in M$ and all vectors $X$ in the Lie algebra $\mathfrak{g}$ of $G$, where, as above, $X_{M}$ denotes the vector field induced by $X: X_{M}(x)=\left.\frac{d}{d t}\right|_{t=0}(\exp t X)$. $x$.

If $d \beta$ is a symplectic form then, up to a sign convention, $\Psi_{\beta}$ is a symplectic moment map. If $\alpha$ a contact form then $\Psi_{\alpha}$ is a candidate for a contact moment map. Note however that if $f$ is a $G$-invariant function, then $e^{f} \alpha$ is also a contact form defining the same contact distribution, while clearly $\Psi_{e^{f} \alpha}=e^{f} \Psi_{\alpha}$. That is, this definition of the moment map depends on a particular choice of a contact form and not just on the contact structure.

Fortunately there is also a notion of a contact moment map that doesn't have this problem. Namely, suppose again that a Lie group $G$ acts on a manifold $M$ preserving a co-oriented contact structure $\xi$. The lift of the action of $G$ to the cotangent bundle then preserves a component $\xi_{+}^{\circ}$ of $\xi^{\circ} \backslash 0$. The restriction $\Psi=\left.\Phi\right|_{\xi_{+}^{\circ}}$ of the moment map $\Phi$ for the action of $G$ on $T^{*} M$ to $\xi_{+}^{\circ}$ depends only on the action of the group and on the contact structure. Moreover, since $\Phi: T^{*} M \rightarrow \mathfrak{g}^{*}$ is 
given by the formula

$$
\langle\Phi(q, p), X\rangle=\left\langle p, X_{M}(q)\right\rangle
$$

for all $q \in M, p \in T_{q}^{*} M$ and $X \in \mathfrak{g}$, we see that if $\alpha$ is any invariant contact form with $\operatorname{ker} \alpha=\xi$ and $\alpha(M) \subset \xi_{+}^{\circ}$ then $\left\langle\alpha^{*} \Psi(q), X\right\rangle=$ $\left\langle\alpha^{*} \Phi(q), X\right\rangle=\left\langle\alpha_{q}, X_{M}(q)\right\rangle=\left\langle\Psi_{\alpha}(q), X\right\rangle$. Thus $\Psi \circ \alpha=\Psi_{\alpha}$, that is, $\Psi=\left.\Phi\right|_{\xi_{+}^{\circ}}$ is a "universal" moment map.

There is another reason why the universal moment map $\Psi: \xi_{+}^{\circ} \rightarrow \mathfrak{g}^{*}$ is a more natural notion of the moment map than the one given by (2.1). The vector fields induced by the action of $G$ preserving the contact distribution $\xi$ are contact. The space of contact vector fields is isomorphic to the space of sections of the bundle $T M / \xi \rightarrow M$ (a choice of a contact form identifies $T M / \xi$ with $M \times \mathbb{R}$ and contact vector fields with functions). Thus a contact group action gives rise to a linear map

$$
\mathfrak{g} \rightarrow \Gamma(T M / \xi), \quad X \mapsto X_{M} \bmod \xi .
$$

The moment map should be the transpose of the map (2.2). The total space of the bundle $(T M / \xi)^{*}$ naturally maps into the space dual to the space of sections $\Gamma(T M / \xi)$ :

$$
(T M / \xi)^{*} \ni \eta \mapsto(s \mapsto\langle\eta, s(\pi(\eta))\rangle),
$$

where $\pi:(T M / \xi)^{*} \rightarrow M$ is the projection and $\langle\cdot, \cdot\rangle$ is the paring between the corresponding fibers of $(T M / \xi)^{*}$ and $T M / \xi$. In other words, the transpose $\Psi:(T M / \xi)^{*} \rightarrow \mathfrak{g}^{*}$ of $(2.2)$ should be given by

$$
\langle\Psi(\eta), X\rangle=\left\langle\eta, X_{M}(\pi(\eta)) \bmod \xi\right\rangle
$$

Under the identification $\xi^{\circ} \simeq(T M / \xi)^{*}$, the equation above becomes

$$
\langle\Psi(q, p), X\rangle=\left\langle p, X_{M}(q)\right\rangle
$$

for all $q \in M, p \in \xi_{q}^{\circ}$ and $X \in \mathfrak{g}$, which exactly the definition of $\Psi$ given earlier as the restriction of the moment map for the lifted action of $G$ on the cotangent bundle $T^{*} M$. Thus part of the above discussion can be summarized as

Proposition 2.8. Let $(M, \xi)$ be a co-oriented contact manifold with an action of a Lie group $G$ preserving the contact distribution and its coorientation. Suppose there exists an invariant 1-form $\alpha$ with $\operatorname{ker} \alpha=\xi$ and $\alpha(M) \subset \xi_{+}^{\circ}$ (c.f. Lemma 2.6). Then the $\alpha$-moment map $\Psi_{\alpha}$ for the action of $G$ on $(M, \alpha)$ and the moment map $\Psi$ for the action of $G$ on the symplectization $\xi_{+}^{\circ}$ are related by

$$
\Psi \circ \alpha=\Psi_{\alpha} .
$$


Here $\xi_{+}^{\circ}$ is the component of $\xi \backslash 0$ containing the image of $\alpha: M \rightarrow \xi^{\circ}$.

REMARK 2.9. We will refer to $\Psi: \xi_{+}^{\circ} \rightarrow \mathfrak{g}^{*}$ as the moment map for the action of a Lie group $G$ on a co-oriented contact manifold $(M, \xi=$ ker $\alpha$ ), that is, as the contact moment map. It is easy to show that $\Psi$ is $G$-equivariant with respect to the given action of $G$ on $M$ and the coadjoint action of $G$ on $\mathfrak{g}^{*}$. Hence for any invariant contact form $\alpha$ the corresponding $\alpha$-moment map $\Psi_{\alpha}: M \rightarrow \mathfrak{g}^{*}$ is also $G$-equivariant.

Later in the paper we will need a version of contact reduction due to Albert $[\mathbf{A}]$ and, independently, to Geiges $[\mathbf{G e}]$ :

Lemma 2.10 (Contact quotients). Suppose a Lie group $G$ acts on a manifold $M$ preserving a 1-form $\beta$. Let $\Psi_{\beta}: M \rightarrow \mathfrak{g}^{*}$ denote the corresponding moment map. Suppose $\Psi_{\beta}^{-1}(0)$ is a manifold and suppose that $G$ acts freely and properly on $\Psi_{\beta}^{-1}(0)$. Then $\beta$ descends to a 1-form $\beta_{0}$ on $M_{0}:=\Psi_{\beta}^{-1}(0) / G$.

If $\beta$ is contact than $\beta_{0}$ is contact as well. Moreover the manifold $M_{0}$ and the contact structure on $M_{0}$ defined by $\beta_{0}$ depends only on the contact structure defined by $\beta$ and not on the form $\beta$ itself.

Sketch of proof. It's easy to see that $\left.\beta\right|_{\Psi_{\beta}^{-1}(0)}$ is basic $^{1}$ and hence descends. For a proof that $\beta_{0}$ is contact if $\beta$ is contact see $[\mathbf{A}]$ or $[\mathbf{G e}]$. Note that the zero level set $\Psi_{\beta}^{-1}(0)$ is the set of points of $M$ where $G$ orbits are tangent to the contact distribution $\operatorname{ker} \beta$. Thus $M_{0}$ depends only on $\operatorname{ker} \beta$. It is not hard to see that $\operatorname{ker} \beta_{0}$ depends only on $\operatorname{ker} \beta$ as well.

We now begin the study of contact toric manifolds.

Definition 2.11. An action of a torus $G$ on a contact manifold $(M, \xi)$ is completely integrable if it is effective, preserves the contact structure $\xi$ and if $2 \operatorname{dim} G=\operatorname{dim} M+1$.

A contact toric $G$-manifold is a co-oriented contact manifold $(M, \xi)$ with a completely integrable action of a torus $G$ (but see Remark 2.13 below).

Note that if an action of a torus $G$ on $(M, \xi)$ is completely integrable, then the action of $G$ on a component $\xi_{+}^{\circ}$ of $\xi^{\circ} \backslash 0$ is a completely integrable Hamiltonian action and thus $\xi_{+}^{\circ}$ is a symplectic toric manifold

\footnotetext{
${ }^{1}$ that is, the form $\left.\beta\right|_{\Psi_{\beta}^{-1}(0)}$ is $G$-invariant and its contraction with any vector field induced by an element $X$ in the Lie algebra of $G$ is zero
} 
(for more information on symplectic toric manifolds and orbifolds see $[\mathbf{D}]$ and $[\mathbf{L T}])$.

Lemma 2.12. Suppose an action of a torus $G$ on a contact manifold $(M, \xi)$ is completely integrable. Then zero is not in the image of the contact moment map $\Psi: \xi_{+}^{\circ} \rightarrow \mathfrak{g}^{*}$.

Proof. Suppose not. Then for some point $x \in M$ the orbit $G \cdot x$ is tangent to the contact distribution $\xi$. Therefore the tangent space $\zeta_{x}:=T_{x}(G \cdot x)$ is isotropic in the symplectic vector space $\left(\xi_{x}, \omega_{x}\right)$ where $\omega_{x}=\left.d \alpha_{x}\right|_{\xi}$ and $\alpha$ is a $G$-invariant contact form with $\operatorname{ker} \alpha=\xi$ and $\alpha(M) \subset \xi_{+}^{\circ}$.

We now argue that this forces the action of $G$ not to be effective. More precisely we argue that the slice representation of the connected component of identity $H$ of the isotropy group $G_{x}$ of the point $x$ is not effective. The group $H$ acts on $\xi_{x}$ preserving the symplectic form $\omega_{x}$ and preserving $\zeta_{x}$. Since $\zeta_{x}$ is isotropic, $\xi_{x}=\left(\zeta_{x}^{\omega} / \zeta_{x}\right) \oplus\left(\zeta_{x} \times \zeta_{x}^{*}\right)$ as a symplectic representation of $H$. Here $\zeta_{x}^{\omega}$ denotes the symplectic perpendicular to $\zeta_{x}$ in $\left(\xi_{x}, \omega_{x}\right)$. Note that since $G$ is a torus, the action of $H$ on $\zeta_{x}$ (and hence on $\zeta_{x}^{*}$ ) is trivial.

Observe next that the dimension of the symplectic vector space $V:=$ $\zeta_{x}^{\omega} / \zeta_{x}$ is $\operatorname{dim} \xi_{x}-2 \operatorname{dim} \zeta_{x}=\operatorname{dim} M-1-2(\operatorname{dim} G-\operatorname{dim} H)=(\operatorname{dim} M-$ 1) $-(\operatorname{dim} M+1)+2 \operatorname{dim} H=2 \operatorname{dim} H-2$. On the other hand, since $H$ is a compact connected Abelian group acting symplectically on $V$, its image in the group of symplectic linear transformations $\operatorname{Sp}(V)$ lies in a maximal torus $T$ of a maximal compact subgroup of $\operatorname{Sp}(V)$. Since the maximal compact subgroup of $\operatorname{Sp}(V)$ is the unitary group $U(n)$, $n=\operatorname{dim} V / 2$, the dimension of $T$ is $n=\operatorname{dim} H-1$. Therefore the representation of $H$ on $V$ (and hence of $G_{x}$ ) is not faithful. Since the fiber at $x$ of the normal bundle of $G \cdot x$ in $M$ is $T_{x} M / \xi_{x} \oplus \xi_{x} / \zeta_{x} \simeq$ $\mathbb{R} \oplus V \oplus \zeta_{x}^{*}$, the slice representation of $G_{x}$ is not faithful. Consequently the action of $G$ is not faithful in a neighborhood of an orbit $G \cdot x$. Contradiction.

Suppose $(M, \xi)$ is a contact toric $G$-manifold. Fix an inner product on the Lie algebra $\mathfrak{g}$ of $G$ and thereby on the dual space $\mathfrak{g}^{*}$. There exists then a unique $G$-invariant contact form $\alpha$ defining $\xi$ and its coorientation and furthermore satisfying the normalizing condition that $\left\|\Psi_{\alpha}(x)\right\|=1$ at all $x \in M$ (if $\alpha^{\prime}$ is any contact form defining $\xi$ let $\alpha_{x}=\frac{1}{\left\|\Psi_{\alpha^{\prime}}(x)\right\|} \alpha_{x}^{\prime}$ for $x \in M$; by Lemma 2.12 this makes sense). 
REMARK 2.13. ¿From now on we fix an inner product on a torus $G$. This allows us to normalize contact forms on contact toric $G$-manifolds as above. Also, since the moment map $\Psi_{\alpha}: M \rightarrow \mathfrak{g}^{*}$ contains all the information about the action of $G$ on $(M, \xi=\operatorname{ker} \alpha)$ we can and will think of a contact toric $G$-manifold as a triple $\left(M, \alpha, \Psi_{\alpha}\right)$ where $\alpha$ is normalized so that $\left\|\Psi_{\alpha}(x)\right\|=1$ for all $x \in M$.

Definition 2.14. Let $(M, \xi)$ be a co-oriented contact manifold with an action of a Lie group $G$ preserving the contact structure $\xi$ and its co-orientation. Let $\Psi: \xi_{+}^{\circ} \rightarrow \mathfrak{g}^{*}$ denote the corresponding moment map. We define the moment cone $C(\Psi)$ to be the set

$$
C(\Psi):=\Psi\left(\xi_{+}^{\circ}\right) \cup\{0\} .
$$

Note that if $\alpha$ is a $G$-invariant contact form with $\xi=\operatorname{ker} \alpha$ and $\alpha(M) \subset$ $\xi_{+}^{\circ}$, then

$$
C(\Psi)=\left\{t f \mid f \in \Psi_{\alpha}(M), t \in[0, \infty)\right\},
$$

where $\Psi_{\alpha}: M \rightarrow \mathfrak{g}^{*}$ denote the $\alpha$-moment map.

Definition 2.15. Two contact toric $G$-manifolds $\left(M, \alpha, \Psi_{\alpha}\right)$ and $\left(M, \alpha^{\prime}, \Psi_{\alpha^{\prime}}\right)$ are isomorphic if there exists a $G$-equivariant co-orientation preserving contactomorphism $\varphi: M \rightarrow M^{\prime}$. We will refer to such a map $\varphi$ as an isomorphism between $\left(M, \alpha, \Psi_{\alpha}\right)$ and $\left(M, \alpha^{\prime}, \Psi_{\alpha^{\prime}}\right)$.

We denote the group of isomorphisms of $\left(M, \alpha, \Psi_{\alpha}\right)$ by $\operatorname{Iso}\left(M, \alpha, \Psi_{\alpha}\right)=$ $\operatorname{Iso}(M)$

REMARK 2.16. Note that if $\varphi: M \rightarrow M^{\prime}$ is an isomorphism of two contact toric $G$-manifolds $\left(M, \alpha, \Psi_{\alpha}\right)$ and $\left(M, \alpha^{\prime}, \Psi_{\alpha^{\prime}}\right)$, then $\varphi^{*} \alpha^{\prime}=e^{f} \alpha$ for some $G$-invariant function $f \in C^{\infty}(M)$. Consequently $\varphi^{*} \Psi_{\alpha^{\prime}}=$ $e^{f} \Psi_{\alpha}$. But for any $x \in M, 1=\left\|\Psi_{\alpha^{\prime}}(\varphi(x))\right\|=\left\|e^{f(x)} \Psi_{\alpha}(x)\right\|=e^{f(x)} 1=$ $e^{f(x)}$. Thus $\varphi^{*} \alpha^{\prime}=\alpha$ and $\varphi^{*} \Psi_{\alpha^{\prime}}=\Psi_{\alpha}$.

Definition 2.17 (Good cones). Let $\mathfrak{g}^{*}$ be the dual of the Lie algebra of a torus $G$. Recall that a subset $C \subset \mathfrak{g}^{*}$ is a rational polyhedral cone if there exists a finite set of vectors $\left\{v_{i}\right\}$ in the integral lattice $\mathbb{Z}_{G}$ of $G$ such that

$$
C=\bigcap\left\{\eta \in \mathfrak{g}^{*} \mid\left\langle\eta, v_{i}\right\rangle \geq 0\right\} .
$$

Of course it is no loss of generality to assume that the set $\left\{v_{i}\right\}$ is minimal, i.e., that for any index $j$

$$
C \neq \bigcap_{i \neq j}\left\{\eta \in \mathfrak{g}^{*} \mid\left\langle\eta, v_{i}\right\rangle \geq 0\right\},
$$


and that each vector $v_{i}$ is primitive, i.e., $s v_{i} \notin \mathbb{Z}_{G}$ for $s \in(0,1)$. Therefore we make these two assumptions.

A rational polyhedral cone $C=\bigcap_{i=1}^{N}\left\{\eta \in \mathfrak{g}^{*} \mid\left\langle\eta, v_{i}\right\rangle \geq 0\right\},\left\{v_{i}\right\} \subset$ $\mathbb{Z}_{G}$ with non-empty interior is $\operatorname{good}^{2}$ if the annihilator of a linear span of a codimension $k$ space, $0<k<\operatorname{dim} G$ is the Lie algebra of a subtorus $H$ of $G$ and the normals to the face form a basis of the integral lattice $\mathbb{Z}_{H}$ of $H$. That is, if

$$
\{0\} \neq C \cap \bigcap_{j=1}^{k}\left\{\eta \in \mathfrak{g}^{*} \mid\left\langle\eta, v_{i_{j}}\right\rangle \geq 0\right\}
$$

is a face of $C$ for some $\left\{i_{1}, \ldots, i_{k}\right\} \subset\{1, \ldots, N\}$ then

$$
\left\{\sum_{j=1}^{k} a_{j} v_{i_{j}} \mid a_{j} \in \mathbb{R}\right\} \cap \mathbb{Z}_{G}=\left\{\sum_{j=1}^{k} m_{j} v_{i_{j}} \mid m_{j} \in \mathbb{Z}\right\}
$$

and $\left\{v_{i_{j}}\right\}$ is independent over $\mathbb{Z}$.

We can now state the main classification result of the paper:

Theorem 2.18. Compact connected contact toric (c.c.c.t.) G-manifolds $\left(M, \alpha, \Psi_{\alpha}: M \rightarrow \mathfrak{g}^{*}\right)$ are classified as follows.

1. Suppose $\operatorname{dim} M=3$ and the action of $G=\mathbb{T}^{2}$ is free. Then $M$ is a principal G-bundle over $S^{1}$, hence is diffeomorphic to $\mathbb{T}^{3}=S^{1} \times \mathbb{T}^{2}$. Moreover the contact form $\alpha$ is $\cos n t d \theta_{1}+\sin n t d \theta_{2} \quad\left(\left(t, \theta_{1}, \theta_{2}\right) \in\right.$ $S^{1} \times \mathbb{T}^{2}$ ) for some positive integer $n$.

2. Suppose $\operatorname{dim} M=3$ and the action of $G=\mathbb{T}^{2}$ is not free. Then $M$ is diffeomorphic to a lens space (this includes $S^{1} \times S^{2}$ ) and, as a c.c.c.t. $G$-manifold, $\left(M, \alpha, \Psi_{\alpha}\right)$ is classified by two rational numbers $r, q$ with $0 \leq r<1, r<q$.

3. Suppose $\operatorname{dim} M>3$ and the action of $G$ is free. Then $M$ is a principal $G$-bundle over a sphere $S^{d}, d=\operatorname{dim} G-1$. Moreover each principal $G$-bundle over $S^{d}$ has a unique $G$-invariant contact structure making it a c.c.c.t. G-manifold.

4. Suppose $\operatorname{dim} M>3$ and the action of $G$ is not free. Then the moment cone of $\left(M, \alpha, \Psi_{\alpha}\right)$ is a good cone (cf. Definition 2.17 above).

\footnotetext{
${ }^{2}$ for the purposes of this paper
} 
Conversely, given a good cone $C \subset \mathfrak{g}^{*}$ there is a unique c.c.c.t. $G$ manifold $\left(M, \alpha, \Psi_{\alpha}\right)$ with moment cone $C$.

REMARK 2.19. Since principal $n$-torus bundles over a manifold are in one-to-one correspondence with second cohomology classes of the manifold with coefficients in $\mathbb{Z}^{n}$ and since $H^{2}\left(S^{d}, \mathbb{Z}^{n}\right)=0$ for $d \neq 2$, the only interesting case of the part 3 of the theorem occurs when $\operatorname{dim} G=3$. In this case the theorem asserts that each principal $\mathbb{T}^{3}$ bundle over the 2-sphere (there are $\mathbb{Z}^{3}$ of them altogether) carries a unique $\mathbb{T}^{3}$-invariant contact structure. These contact manifolds were first constructed by Lutz $[\mathbf{L u}]$. Their symplectization were also explicitly constructed by Bates [Bt].

We will prove Theorem 2.18 over the course of the next four sections.

\section{Local structure of contact toric manifolds}

In this section we define pre-isotropic embeddings (by analogy with pre-Lagrangian embeddings), prove an equivariant version of the tubular neighborhood for pre-isotropic embeddings and write out the local normal form theorem for a contact moment map in a neighborhood of a pre-isotropic orbit. The pre-isotropic embedding theorem uses an equivariant relative Darboux theorem for submanifolds of contact manifolds. We include its proof for completeness. The local normal form theorem is then applied to contact toric manifolds. Many of the results of this section are not original; similar results have appeared elsewhere, such as in [BaM1, BaM2].

We start with a definition of a pre-isotropic embedding, the motivation for which comes from Lemma 3.7 below. Note that given an embedding $\iota: N \rightarrow M$ we do not distinguish between the vector bundles over $N$ and over $\iota(N)$.

Definition 3.1. Let $(M, \xi=\operatorname{ker} \alpha)$ be a co-oriented contact manifold. An embedded submanifold $N \hookrightarrow M$ is pre-isotropic if

1) $N$ is transverse to the contact distribution $\xi$ and

2) the distribution $\zeta=T N \cap \xi$ is isotropic in the conformal symplectic vector bundle $(\xi,[\omega])$ where $[\omega]$ is the conformal class of $\left.d \alpha\right|_{\xi}$.

REMARK 3.2. Note that $\zeta=\operatorname{ker}\left(\iota^{*} \alpha\right)$. Note also that if $\alpha^{\prime}=e^{f} \alpha$, $f \in C^{\infty}(M)$, is another contact form defining the contact structure $\xi$ then $\iota^{*} \alpha^{\prime}=e^{\iota^{*} f} \iota^{*} \alpha$ and $\left.d \alpha^{\prime}\right|_{\xi}=e^{f}\left(\left.d \alpha\right|_{\xi}\right)$. 
Definition 3.3. Let $\iota: N \hookrightarrow(M, \xi=\operatorname{ker} \alpha)$ be a pre-isotropic embedding. We define the characteristic distribution of the embedding $\iota$ to be the co-oriented distribution $\zeta=T N \cap \xi$. Equivalently we can think of $\zeta$ as the conformal class $\left[\iota^{*} \alpha\right]$ of 1 -forms. We define the conformal symplectic normal bundle $\left(E,\left[\omega_{E}\right]\right)$ of the embedding by $E=\zeta^{\omega} / \zeta$ where $\zeta^{\omega}$ is the symplectic perpendicular to $\zeta$ in the conformal symplectic vector bundle $\left(\xi,[\omega]=\left[\left.d \alpha\right|_{\xi}\right]\right)$ and $\left[\omega_{E}\right]$ is the conformal class of symplectic structures induced on $E$ by $[\omega]$.

Remark 3.4. Suppose $\iota: N \hookrightarrow(M, \xi=\operatorname{ker} \alpha)$ is a pre-isotropic embedding. Suppose further that a Lie group $G$ acts on $N$ and $M$ preserving the contact form $\alpha$ and making the embedding $\iota$ equivariant. Then $G$ preserves the characteristic distribution $\zeta$ and acts on the conformal symplectic normal bundle $E$ preserving the symplectic structure $\omega_{E}$ and its conformal class.

Theorem 3.5 (Uniqueness of pre-isotropic embeddings). A pre-isotropic embedding is uniquely determined by its characteristic distribution and its conformal symplectic normal bundle.

More specifically suppose $\left(M_{j}, \xi_{j}=\operatorname{ker} \alpha_{j}\right), j=1,2$ are two contact manifolds and $\iota_{j}: N \rightarrow\left(M_{j}, \xi_{j}\right), j=1,2$ are two pre-isotropic embeddings such that

$$
\iota_{1}^{*} \alpha_{1}=e^{f} \iota_{2}^{*} \alpha_{2}
$$

and

$$
\left(E_{1}, \omega_{1}\right) \simeq\left(E_{2}, e^{h} \omega_{2}\right) \text { as symplectic vector bundles, }
$$

where $f, h \in C^{\infty}(N)$ are two functions and $\left(E_{1},\left[\omega_{1}\right]\right)$ and $\left(E_{2},\left[\omega_{2}\right]\right)$ are the conformal symplectic normal bundles of the embeddings.

Then there exist neighborhoods $U_{j}$ of $\iota_{j}(N)$ in $M_{j}(j=1,2)$ and a diffeomorphism $\varphi: U_{1} \rightarrow U_{2}$ such that $\iota_{2}=\varphi \circ \iota_{1}$ and $\varphi^{*} \alpha_{2}=e^{g} \alpha_{1}$ for some $g \in C^{\infty}\left(U_{1}\right)$.

Moreover if a Lie group $G$ acts properly on $N, M_{1}, M_{2}$ making the embeddings $\iota_{j} G$-equivariant and if the action preserves the contact structures, then we may choose the neighborhoods $U_{1}, U_{2}$ to be $G$-invariant and the map $\varphi$ to be G-equivariant.

The proof of Theorem 3.5 relies on the following observation.

Theorem 3.6 (Equivariant relative Darboux theorem). Let $N \hookrightarrow M$ be an embedded closed submanifold. Suppose there exist on $M$ two contact structures $\xi^{0}=\operatorname{ker} \alpha^{0}$ and $\xi^{1}=\operatorname{ker} \alpha^{1}\left(\alpha^{0}, \alpha^{1}\right.$ are 1-forms) and a function $f \in C^{\infty}(M)$ such that

$$
\alpha_{x}^{0}=e^{f(x)} \alpha_{x}^{1} \quad \text { for all } x \in N .
$$


Then there exist neighborhoods $U_{0}, U_{1}$ of $N$ in $M$ and a diffeomorphism $\varphi: U_{0} \rightarrow U_{1}$ such that $\left.\varphi\right|_{N}=i d_{N}$ and $\varphi^{*} \alpha^{1}=e^{h} \alpha^{0}$ for some $h \in C^{\infty}\left(U_{0}\right)$.

Moreover, if a Lie group $G$ acts properly on $M$ preserving $N$ and the two contact forms $\alpha^{0}, \alpha^{1}$, then we can choose the neighborhoods $U_{0}, U_{1}$ to be $G$-invariant and arrange for the map $\varphi$ above to be $G$-equivariant.

Proof. Consider the family of $G$-invariant 1-forms $\alpha^{t}=t \alpha^{1}+(1-t) \alpha^{0}$, $t \in[0,1]$. For all $x \in N$ and all $t \in[0,1]$ we have $\operatorname{ker} \alpha_{x}^{t}=\xi_{x}^{0}=\xi_{x}^{1}$ and $\left.d \alpha_{x}^{t}\right|_{\operatorname{ker} \alpha_{x}^{t}}$ is nondegenerate. Therefore the forms $\alpha^{t}$ are contact in a neighborhood of $N$ for all $t$. It is no loss of generality to assume that this neighborhood is all of $M$.

Denote the Reeb vector field of $\alpha^{t}$ by $Y_{t}$. Since the Reeb vector field is uniquely defined by $\alpha^{t}\left(Y_{t}\right)=1, \iota\left(Y_{t}\right) d \alpha^{t}=0$ and since $\alpha^{t}$ is $G$-invariant, $Y_{t}$ is $G$-invariant as well.

Define a time dependent vector field $X_{t}$ tangent to the contact distribution $\xi^{t}=\operatorname{ker} \alpha^{t}$ by

$$
X_{t}=\left(\left.d \alpha^{t}\right|_{\xi^{t}}\right)^{-1}\left(-\left.\dot{\alpha}^{t}\right|_{\xi^{t}}\right)
$$

where $\dot{\alpha}^{t}=\frac{d}{d t} \alpha^{t}$ is the derivative with respect to $t$. Clearly $X_{t}$ is $G$-invariant. Note that $X_{t}(x)=0$ for all $x \in N$. This is because $-\left.\dot{\alpha}_{x}^{t}\right|_{\xi_{x}^{t}}=\left.\left(\alpha_{x}^{0}-\alpha_{x}^{1}\right)\right|_{\xi_{x}^{t}}=\left.\left(e^{f(x)}-1\right) \alpha_{x}^{1}\right|_{\xi_{x}^{1}}=0$ for $x \in N$. We claim that the Lie derivative of $\alpha^{t}$ with respect to $X_{t}$ satisfies

$$
L_{X_{t}} \alpha^{t}=\dot{\alpha}^{t}\left(Y_{t}\right) \alpha^{t}-\dot{\alpha}^{t} .
$$

Indeed, since $\alpha^{t}\left(X_{t}\right)=0, L_{X_{t}} \alpha^{t}=\iota\left(X_{t}\right) d \alpha^{t}$.

By definition of $X_{t},\left.\left(\iota\left(X_{t}\right) d \alpha^{t}\right)\right|_{\xi^{t}}=-\left.\dot{\alpha}^{t}\right|_{\xi^{t}}=\left.\left(\dot{\alpha}^{t}\left(Y_{t}\right) \alpha^{t}-\dot{\alpha}^{t}\right)\right|_{\xi^{t}}$. On the other hand, $\left(\iota\left(X_{t}\right) d \alpha^{t}\right)\left(Y_{t}\right)=0=\dot{\alpha}^{t}\left(Y_{t}\right) 1-\dot{\alpha}^{t}\left(Y_{t}\right)=\dot{\alpha}^{t}\left(Y_{t}\right) \alpha^{t}\left(Y_{t}\right)-$ $\dot{\alpha}^{t}\left(Y_{t}\right)$. This proves (3.1). Hence

$$
L_{X_{t}} \alpha^{t}+\dot{\alpha}^{t}=\dot{\alpha}^{t}\left(Y_{t}\right) \alpha^{t}
$$

Denote the isotopy generated by $X_{t}$ by $\varphi_{t}$. Since $X_{t}$ vanishes on $N$, $\varphi_{t}$ is defined for all $t \in[0,1]$ on a neighborhood of $N$. Since $X_{t}$ is $G$-invariant, the isotopy is $G$-equivariant. Let $g_{t}=\varphi_{t}^{*}\left(\dot{\alpha}^{t}\left(Y_{t}\right)\right)$. Then $\frac{d}{d t}\left(\varphi_{t}^{*} \alpha^{t}\right)=\varphi_{t}^{*}\left(L_{X_{t}} \alpha^{t}+\dot{\alpha}^{t}\right)=g_{t}\left(\varphi_{t}^{*} \alpha^{t}\right)$ by equation (3.2). Therefore $\varphi_{t}^{*} \alpha^{t}=\left(e^{\int_{0}^{t} g_{s} d s}\right) \varphi_{0}^{*} \alpha^{0}$. In particular,

$$
\varphi_{1}^{*} \alpha^{1}=e^{h} \alpha^{0}
$$

where $h=\int_{0}^{1} g_{s} d s \in C^{\infty}(M)$. Note also that since $X_{t}$ is zero at the points of $N, \varphi_{t}$ fixes $N$ pointwise. 
Proof of Theorem 3.5. Suppose $\iota: N \rightarrow(M, \xi=\operatorname{ker} \alpha)$ is a $G$-equivariant pre-isotropic embedding. Choose a $G$-invariant almost complex structure $J$ on $\xi$ compatible with $\omega=\left.d \alpha\right|_{\xi}$. This gives us a $G$-invariant inner product on the vector bundle $\xi \rightarrow M$. Extend it to a $G$-invariant Riemannian metric $g$ on $M$ by, say, declaring the Reeb vector field $Y$ of the invariant contact form $\alpha$ to be of unit length and orthogonal to $\xi$ (as remarked previously, since $\alpha$ is $G$-invariant and the Reeb vector field $Y$ is uniquely determined by $\alpha, Y$ is $G$-invariant).

By construction of $J, \zeta:=\operatorname{ker}\left(\left.\alpha\right|_{N}\right)$ and $J \zeta$ are $g$-perpendicular and $\zeta \oplus J \zeta$ is a symplectic subbundle of $(\xi, \omega)$. Let $E$ be the $\omega$-perpendicular to $\zeta \oplus J \zeta$ in $\xi$. The bundle $E$ is also $g$-perpendicular to $\zeta \oplus J \zeta ; E$ is isomorphic to the conformal symplectic normal bundle of the embedding $\iota$. We therefore have a $G$-equivariant direct sum decomposition

$$
\left.\xi\right|_{N}=\zeta \oplus J \zeta \oplus E=(T N \cap \xi) \oplus J \zeta \oplus E .
$$

Since $N$ is transverse to $\xi$ it follows that

$$
\left.T M\right|_{N}=T N \oplus J \zeta \oplus E .
$$

Note that the Reeb vector field $Y$ need not be tangent to $N$, and so $J \zeta \oplus E$ need not be $g$-perpendicular to $T N$. Never the less, $J \zeta \oplus E$ is a topological normal bundle for the embedding $\iota: N \rightarrow M$. Therefore the restriction of the $g$-exponential map $\exp : T M \rightarrow M$ to $J \zeta \oplus E$ gives an open $G$-equivariant embedding of a neighborhood of the zero section $N \hookrightarrow J \zeta \oplus E$ into $M$; the embedding is identity on $N$.

Since $\zeta$ is isotropic in $(\xi, \omega)$, the map $J \zeta \rightarrow \zeta^{*}$ defined by $v \mapsto$ $\left.\omega(v, \cdot)\right|_{\zeta}$ is an isomorphism. By composing the inverse of this map with $\exp$ we get a $G$-equivariant map $\psi: \zeta^{*} \oplus E \rightarrow M$, which has the following properties. The map $\psi$ is the identity on $N$. It is an open embedding on a sufficiently small neighborhood of $N$ in $\zeta^{*} \oplus E$. For any point $(x, 0,0)$ in the zero section of $\zeta^{*} \oplus E$ we have

$$
\operatorname{ker}\left(\psi^{*} \alpha\right)_{(x, 0,0)}=\zeta_{x} \oplus \zeta_{x}^{*} \oplus E_{x}
$$

and

$$
\left.d\left(\psi^{*} \alpha\right)_{(x, 0,0)}\right|_{\operatorname{ker}\left(\psi^{*} \alpha\right)_{(x, 0,0)}}=\omega_{\zeta_{x} \oplus \zeta_{x}^{*}} \oplus\left(\omega_{E}\right)_{x},
$$

where $\omega_{\zeta_{x} \oplus \zeta_{x}^{*}}$ denotes the canonical symplectic form on $\zeta_{x} \oplus \zeta_{x}^{*}$ and $\left(\omega_{E}\right)_{x}=\left.d \alpha_{x}\right|_{E_{x}}$.

Now consider two pre-isotropic embeddings $\iota_{j}: N \rightarrow\left(M_{j}, \zeta_{j}\right), j=$ 1,2 satisfying the hypotheses of the theorem. Since $\iota_{1}^{*} \alpha_{1}=e^{f} \iota_{2}^{*} \alpha_{2}$, $\operatorname{ker} \iota_{1}^{*} \alpha_{1}=\operatorname{ker} \iota_{2}^{*} \alpha_{2}$. Denote this distribution by $\zeta$. Let $\sigma: E_{1} \rightarrow E_{2}$ denote the vector bundle isomorphism with $\sigma^{*} \omega_{2}=e^{h} \omega_{1}$. The map 
$\tau=i d \oplus \sigma: \zeta^{*} \oplus E_{1} \rightarrow \zeta^{*} \oplus E_{2}$ has the property that at the points $(x, 0,0)$ of the zero section $N$

$$
d \tau_{(x, 0,0)}=\left.i d_{T_{x} N} \oplus i d_{\zeta_{x}^{*}} \oplus \sigma\right|_{\left(E_{1}\right)_{x}} .
$$

Consider the two $G$-equivariant maps $\psi_{j}: \zeta_{j}^{*} \oplus E_{j} \rightarrow M, j=1,2$ given by the construction at the beginning of the proof. We have, for any point $x \in N$,

$$
\begin{aligned}
\operatorname{ker}\left(\left(\psi_{2} \circ \tau\right)^{*} \alpha_{2}\right)_{(x, 0,0)} & =\left(d \tau_{(x, 0,0)}\right)^{-1}\left(\zeta_{x} \oplus \zeta_{x}^{*} \oplus\left(E_{2}\right)_{x}\right) \\
& =\zeta_{x} \oplus \zeta_{x}^{*} \oplus\left(E_{1}\right)_{x} \\
& =\operatorname{ker}\left(\psi_{1}^{*} \alpha_{1}\right)_{(x, 0,0)}
\end{aligned}
$$

Also

$$
\left.d\left(\psi_{1}^{*} \alpha_{1}\right)_{(x, 0,0)}\right|_{\operatorname{ker}\left(\psi_{1}^{*} \alpha_{1}\right)_{(x, 0,0)}}=\omega_{\zeta_{x} \oplus \zeta_{x}^{*}} \oplus\left(\omega_{1}\right)_{x}
$$

while

$\left.d\left(\tau^{*} \psi_{2}^{*} \alpha_{2}\right)_{(x, 0,0)}\right|_{\operatorname{ker}\left(\psi_{1}^{*} \alpha_{1}\right)_{(x, 0,0)}}=\left(i d_{\zeta} \oplus i d_{\zeta^{*}}\right)^{*} \omega_{\zeta_{x} \oplus \zeta_{x}^{*}} \oplus \sigma^{*}\left(\omega_{2}\right)_{x}=\omega_{\zeta_{x} \oplus \zeta_{x}^{*}} \oplus\left(e^{h} \omega_{1}\right)_{x}$.

We now apply the equivariant relative Darboux theorem to $N \hookrightarrow$ $\zeta^{*} \oplus E_{1}, \psi_{1}^{*} \alpha_{1}$ and $\tau^{*} \psi_{2}^{*} \alpha_{2}$ to obtain a $G$-equivariant diffeomorphism $F: V_{1} \rightarrow V_{2}$ with $\left.F\right|_{N}=i d_{N}, F^{*} \tau^{*} \psi_{2}^{*} \alpha_{2}=e^{f}\left(\psi_{1}^{*} \alpha_{1}\right)$ for some neighborhoods $V_{1}, V_{2}$ of the zero section and some function $f$. The theorem follows.

Lemma 3.7. Let $\Psi_{\alpha}: M \rightarrow \mathfrak{g}^{*}$ be the $\alpha$-moment map for an action of a torus $G$ on a contact manifold $(M, \alpha)$. Suppose for some point $x$ we have $\Psi_{\alpha}(x) \neq 0$. Then the orbit $G \cdot x$ is pre-isotropic in $(M, \xi=\operatorname{ker} \alpha)$.

Proof. Since the contact distribution $\xi$ is of codimension 1, in order to show that the orbit $G \cdot x$ is transverse to $\xi$, it is enough to prove that there is a vector $X \in \mathfrak{g}$ such that $X_{M}(x) \notin \xi_{x}$, i.e., such that $0 \neq \alpha_{x}\left(X_{M}(x)\right)=\left\langle\Psi_{\alpha}(x), X\right\rangle$. But the latter is exactly the condition that $\Psi_{\alpha}(x) \neq 0$.

Next note that the fiber of the characteristic distribution $\zeta$ at $x$ is

$$
\zeta_{x}=T_{x}(G \cdot x) \cap \xi_{x}=\left\{X_{M}(x) \mid 0=\alpha_{x}\left(X_{M}(x)\right)=\left\langle\Psi_{\alpha}(x), X\right\rangle\right\} .
$$

Let $\mathfrak{k}=\left\{X \in \mathfrak{g} \mid\left\langle\Psi_{\alpha}(x), X\right\rangle=0\right\}$. Since $\mathfrak{g}$ is Abelian, $\mathfrak{k}$ is a subalgebra. Consequently $\zeta$ is an integrable distribution. Since the leaves of the foliation defined by $\zeta$ are tangent to the contact structure, $\zeta$ is an isotropic subbundle of the symplectic vector bundle $(\xi, \omega)$, where as usual $\omega=\left.d \alpha\right|_{\xi}$. 
Definition 3.8. Let $(M, \xi=\operatorname{ker} \alpha)$ be a contact manifold with an action of a torus $G$ preserving the contact form $\alpha$ and let $\Psi_{\alpha}: M \rightarrow \mathfrak{g}^{*}$ be the corresponding moment map. Suppose a point $x \in M$ is such that $\Psi_{\alpha}(x) \neq 0$. Let $\zeta_{x}$ denotes the fiber at $x$ of the characteristic distribution of the pre-isotropic embedding $G \cdot x \hookrightarrow(M, \xi)$ and let $\zeta_{x}^{\omega}$ denote its symplectic perpendicular in $\left(\xi, \omega=\left.d \alpha\right|_{\xi}\right)$. We define the symplectic slice at $x$ for the action of $G$ on $(M, \xi)$ to be the conformal symplectic vector space $V=\zeta_{x}^{\omega} / \zeta_{x}$ with the conformal symplectic structure $\left[\omega_{V}\right]$ induced by $[\omega]$. We refer to the symplectic representation of the isotropy group $G_{x}$ on $\left(V, \omega_{V}\right)$ as the symplectic slice representation.

We define the characteristic subalgebra of the embedding $G \cdot x \hookrightarrow$ $(M, \xi)$ to be $\mathfrak{k}:=\left(\mathbb{R} \Psi_{\alpha}(x)\right)^{\circ}$. Note that $\zeta_{x}=\mathfrak{k} / \mathfrak{g}_{x}$ and that $\mathfrak{k}$ is cooriented.

Lemma 3.9. Let $\left(M_{j}, \xi_{j}=\operatorname{ker} \alpha_{j}\right), j=1,2$, be two contact manifolds with actions of a torus $G$ preserving the contact forms $\alpha_{1}, \alpha_{2}$. Suppose $x_{j} \in M_{j}, j=1,2$ are two points such that

1) $0 \neq \Psi_{\alpha_{1}}\left(x_{1}\right)=\lambda \Psi_{\alpha_{2}}\left(x_{2}\right)$ for some $\lambda>0$ (i.e., the characteristic subalgebras agree as co-oriented subspaces of $\mathfrak{g})$;

2) the isotropy groups are equal: $G_{x_{1}}=G_{x_{2}}$;

3) the symplectic slice representations at $x_{1}$ and $x_{2}$ are isomorphic as symplectic representation up to a conformal factor.

Then there exist $G$-invariant neighborhoods $U_{j}$ of $G \cdot x_{j}$ in $M_{j}, j=1,2$, and a G-equivariant diffeomorphism $\varphi: U_{1} \rightarrow U_{2}$ such that $\varphi^{*} \alpha_{2}=$ $e^{f} \alpha_{1}$ for some function $f$.

Proof. The characteristic distributions and the conformal symplectic normal bundles of the embeddings $\iota_{j}: G \cdot x_{j} \hookrightarrow\left(M_{j}, \xi_{j}\right), j=1,2$, are, respectively,

$$
\zeta_{j}=G \times_{G_{x_{j}}}\left(\mathfrak{k} / \mathfrak{g}_{x_{j}}\right) \quad \text { and } \quad E_{j}=G \times_{G_{x_{j}}} V_{j}
$$

where $\mathfrak{k}=\left(\mathbb{R} \Psi_{\alpha_{1}}\left(x_{1}\right)\right)^{\circ}=\left(\mathbb{R} \Psi_{\alpha_{2}}\left(x_{2}\right)\right)^{\circ}$ and $G_{x_{j}} \rightarrow \operatorname{Sp}\left(V_{j}, \omega_{V_{j}}\right)$ are the symplectic slice representations. The lemma follows from the uniqueness of pre-isotropic embeddings (Theorem 3.5).

Lemma 3.10. Let $(M, \xi=\operatorname{ker} \alpha)$ be a contact manifold with action of a torus $G$ preserving the contact form $\alpha$. Suppose $x \in M$ is such that $\Psi_{\alpha}(x) \neq 0 . \quad$ Let $\mathfrak{k}=\left(\mathbb{R} \Psi_{\alpha}(x)\right)^{\circ}$ be the characteristic subalgebra and 
$G_{x} \rightarrow \operatorname{Sp}\left(V, \omega_{V}\right)$ the symplectic slice representation. Choose splittings

$$
\begin{aligned}
& \mathfrak{g}_{x}^{\circ}=\left(\mathfrak{k} / \mathfrak{g}_{x}\right)^{*} \oplus \mathbb{R} \Psi_{\alpha}(x) \\
& \mathfrak{g}^{*}=\mathfrak{g}_{x}^{\circ} \oplus \mathfrak{g}_{x}^{*}
\end{aligned}
$$

and thereby a splitting

$$
\mathfrak{g}^{*}=\left(\mathfrak{k} / \mathfrak{g}_{x}\right)^{*} \oplus \mathbb{R} \Psi_{\alpha}(x) \oplus \mathfrak{g}_{x}^{*} .
$$

Let $i: \mathfrak{g}_{x}^{*} \hookrightarrow \mathfrak{g}^{*}, j:\left(\mathfrak{k} / \mathfrak{g}_{x}\right)^{*} \hookrightarrow \mathfrak{g}^{*}$ be the corresponding embeddings.

There exists a $G$-invariant neighborhood $U$ of the zero section $G$. $[1,0,0]$ in

$$
N=G \times_{G_{x}}\left(\left(\mathfrak{k} / \mathfrak{g}_{x}\right)^{*} \oplus V\right)
$$

and an open $G$-equivariant embedding $\varphi: U \hookrightarrow M$ with $\varphi([1,0,0])=x$ and a $G$-invariant 1-form $\alpha_{N}$ on $N$ such that

1) $\varphi^{*} \alpha=e^{f} \alpha_{N}$ for some function $f \in C^{\infty}(U)$ and

2) the $\alpha_{N}$-moment map $\Psi_{\alpha_{N}}$ is given by

$$
\Psi_{\alpha_{N}}([a, \eta, v])=\Psi_{\alpha}(x)+j(\eta)+i\left(\Phi_{V}(v)\right)
$$

where $\Phi_{V}: V \rightarrow \mathfrak{g}^{*}$ is the homogeneous moment map for the slice representation.

Consequently,

$\Psi_{\alpha} \circ \varphi([a, \eta, v])=\left(e^{f} \Psi_{\alpha_{N}}\right)([a, \eta, v])=e^{f([a, \eta, v])}\left(\Psi_{\alpha}(x)+j(\eta)+i\left(\Phi_{V}(v)\right)\right)$,

for some $G$-invariant function $f$ on $N$.

Proof. By Lemma 3.9 it is enough to construct on $N=G \times_{G_{x}}\left(\left(\mathfrak{k} / \mathfrak{g}_{x}\right)^{*} \times\right.$ $V)$ a $G$-invariant contact form $\alpha_{N}$ so that the embedding $\iota: G / G_{x} \hookrightarrow$ $\left(N, \operatorname{ker} \alpha_{N}\right), \iota\left(a G_{x}\right)=[a, 0,0]$ is pre-isotropic, the symplectic slice representation at $[1,0,0]$ is $G_{x} \rightarrow \operatorname{Sp}\left(V, \omega_{V}\right)$ and $\Psi_{\alpha_{N}}([1,0,0])=\Psi_{\alpha}(x)$.

We construct $\left(N, \alpha_{N}\right)$ as a contact quotient (see Lemma 2.10). Since $G$ is abelian, both right and left trivializations identify the cotangent bundle $T^{*} G$ with $G \times \mathfrak{g}^{*}$. Consider the hypersurface

$$
\Sigma=G \times\left(\Psi_{\alpha}(x)+j\left(\left(\mathfrak{k} / \mathfrak{g}_{x}\right)^{*}\right)+i\left(\mathfrak{g}_{x}^{*}\right)\right)
$$

in $G \times \mathfrak{g}^{*}=T^{*} G$. Since $\Psi_{\alpha}(x) \neq 0, \Sigma$ is a hypersurface of contact type (the expanding vector field $X$ is generated by dilations $(0, \infty) \times$ $\left.G \times \mathfrak{g}^{*} \ni(t, g, \nu) \mapsto(g, t \nu)\right)$. Consider the action of $G$ on $G \times \mathfrak{g}^{*}$ given by $g \cdot(a, \nu)=(g a, \nu)$ and the action of $G_{x}$ on $G \times \mathfrak{g}^{*}$ given by $b \cdot(a, \nu)=\left(a b^{-1}, \nu\right)$. Both actions preserve $\Sigma, X$ and the tautological 1-form $\alpha_{T^{*} G}$. The action of $G_{x}$ on $V$ preserves the 1-form $\alpha_{V}=\iota(R) \omega_{V}$ where $R$ is the radial vector field on $V$. The diagonal action of $G_{x}$ on 
$\Sigma \times V$ preserves the contact form $\left(\left.\alpha_{T^{*} G}\right|_{\Sigma}\right) \oplus \alpha_{V}$. The corresponding moment map $\Phi: \Sigma \times V \rightarrow \mathfrak{g}_{x}^{*}$ is given by

$$
\Phi\left(\left(a, \Psi_{\alpha}(x)+j(\eta)+i(\mu)\right), v\right)=-\mu+\Phi_{V}(v)
$$

where $\Phi_{V}: V \rightarrow \mathfrak{g}_{x}^{*}$ is the $\alpha_{V}$-moment map. Therefore the reduced space at zero for the action of $G_{x}$ is

$$
\begin{aligned}
N & :=\Phi^{-1}(0) / G_{x} \\
& \simeq\left\{(a, \eta, \mu, v) \in G \times\left(\mathfrak{k} / \mathfrak{g}_{x}\right)^{*} \times \mathfrak{g}_{x}^{*} \times V \mid \mu=\Phi_{V}(v)\right\} / G_{x}=G \times_{G_{x}}\left(\left(\mathfrak{k} / \mathfrak{g}_{x}\right)^{*} \times V\right)
\end{aligned}
$$

and $\left.\alpha_{T^{*} G}\right|_{\Sigma} \oplus \alpha_{V}$ descends to a $G$-invariant contact form $\alpha_{N}$ on $N$.

Note that the moment map for the action of $G$ on $\Sigma \times V$ descends to the $\alpha_{N}$-moment map for the induced action of $G$ on $N$. Hence it is given by the desired formula:

$$
\Psi_{\alpha_{N}}([a, \eta, v])=\Psi_{\alpha}(x)+j(\eta)+i\left(\Phi_{V}(v)\right) .
$$

We will need the following standard fact.

Lemma 3.11. A symplectic representation of a torus has well-defined weights.

Proof. Since the unitary group is the maximal compact subgroup of the symplectic group, given a symplectic representation of a torus $\rho$ : $H \rightarrow \operatorname{Sp}(V, \omega)$ there exists on $V$ an $H$-invariant complex structure $J$ compatible with the symplectic form $\omega$. We define the weights of $\rho$ to be the weights of the complex representation $\rho: H \rightarrow \operatorname{GL}(V, J)$. Since any two $H$-invariant complex structures on $V$ compatible with $\omega$ are homotopic, the weights do not depend on the choice of $J$, i.e., they are well-defined.

The next lemma is taken from $[\mathbf{D}]$.

Lemma 3.12. If $\rho: H \rightarrow S p(V, \omega)$ is a faithful symplectic representation of a compact abelian group $H$ and if $2 \operatorname{dim} H=\operatorname{dim} V$ then $H$ is connected and the weights of $\rho$ form a basis of the weight lattice $\mathbb{Z}_{H}^{*}$ of $H$.

Proof. Arguing as in the proof of Lemma 3.11 we may assume that $\rho$ maps $H$ into the unitary group $U(V)$. Since the dimension of a maximal torus of $U(V)$ is $\frac{1}{2} \operatorname{dim} V$ and since $\rho$ is faithful, $\rho$ maps the identity component of $H$ onto a maximal torus of $U(V)$. Since the centralizer of a maximal torus of $U(V)$ is the torus itself, $H$ is connected. Finally, 
the weights of a maximal torus $T$ of $U(V)$ form a basis of the weight lattice of $T$.

Recall that $G$-orbits in contact toric $G$-manifolds are pre-isotropic (Lemma 3.7 and Lemma 2.12).

Lemma 3.13. Let $\left(M, \alpha, \Psi_{\alpha}: M \rightarrow \mathfrak{g}^{*}\right)$ be a contact toric $G$-manifold. For any point $x \in M$ the symplectic slice representation $\rho: G_{x} \rightarrow$ $\operatorname{Sp}(V)$ is faithful and $\operatorname{dim} G_{x}=\frac{1}{2} \operatorname{dim} V$. Consequently the isotropy group $G_{x}$ is connected. Also the image of the moment map $\Phi_{V}(V)$ for the slice representation $\rho$ has the following properties: the cone $\Phi_{V}(V)$ has $d=\operatorname{dim} G_{x}$ edges; each edge is spanned by a weight of $G_{x}$; these weights form a basis of the integral lattice of $G_{x}$. Hence the cone $\Phi_{V}(V)$ completely determines the slice representation $\rho$.

Proof. Let $\mathfrak{k}=\left(\mathbb{R} \Psi_{\alpha}(x)\right)^{\circ}$ denote the characteristic subalgebra of the pre-isotropic embedding $G \cdot x \hookrightarrow(M, \xi=\operatorname{ker} \alpha)$. By Lemma 3.10 a neighborhood of $G \cdot x$ in $M$ is $G$-equivariantly diffeomorphic to a neighborhood of the zero section in $N=G \times{ }_{G_{x}}\left(\left(\mathfrak{k} / \mathfrak{g}_{x}\right)^{*} \times V\right)$. Since $G$ is abelian, the action of $G_{x}$ on $\left(\mathfrak{k} / \mathfrak{g}_{x}\right)^{*}$ is trivial. Since by assumption the action of $G$ on $M$ is effective, the slice representation of $G_{x}$ on $V$ has to be faithful.

By definition of $V$, the dimension of $V$ is the dimension of the contact distribution minus twice the dimension of the characteristic distribution, i.e., $\operatorname{dim} V=(\operatorname{dim} M-1)-2 \operatorname{dim}\left(\mathfrak{k} / \mathfrak{g}_{x}\right) . \quad$ Now $\operatorname{dim} M-1=$ $2 \operatorname{dim} G-2$ and $\operatorname{dim} \mathfrak{k}=\operatorname{dim} G-1$. Therefore, $\operatorname{dim} V=2 \operatorname{dim} G-2-$ $2\left((\operatorname{dim} G-1)-\operatorname{dim} G_{x}\right)=2 \operatorname{dim} G_{x}$. By Lemma $3.12 G_{x}$ is connected and the weights $\nu_{1}, \ldots \nu_{d}\left(d=\operatorname{dim} G_{x}=\frac{1}{2} \operatorname{dim} V\right)$ of the slice representation $\rho$ form a basis of the weight lattice of $G_{x}$. On the other hand, $\Phi_{V}(V)=\left\{\sum_{i=1}^{d} a_{i} \nu_{i} \mid a_{i} \geq 0\right\}$. The rest of the lemma follows.

As a corollary of the first part of the proof and Lemma 3.10 we get

Theorem 3.14. Let $\left(M, \alpha, \Psi_{\alpha}: M \rightarrow \mathfrak{g}^{*}\right)$ be a c.c.c.t. $G$-manifold normalized so that $\Psi_{\alpha}(M) \subset S\left(\mathfrak{g}^{*}\right)=\left\{\eta \in \mathfrak{g}^{*} \mid\|\eta\|=1\right\}$. Let $x \in$ $M$ be a point, $G_{x}$ be its isotropy group (which is connected). Let $\rho$ : $G_{x} \rightarrow S p\left(V, \omega_{V}\right)$ denote the symplectic slice representation, $\Phi: V \rightarrow \mathfrak{g}^{*}$ denote the corresponding moment map, and let $\mathfrak{k}=\left(\mathbb{R} \Psi_{\alpha}(x)\right)^{\circ}$ be the characteristic subalgebra. Choose the embeddings $i: \mathfrak{g}_{x}^{*} \rightarrow \mathfrak{g}^{*}, j:$ $\left(\mathfrak{k} / \mathfrak{g}_{x}\right)^{*} \rightarrow \mathfrak{g}^{*}$ as in Lemma 3.10 . 
There exists an open embedding $\varphi$ from a neighborhood of the orbit $G / G_{x} \times\{0\} \times\{0\}$ in $G / G_{x} \times\left(\mathfrak{k} / \mathfrak{g}_{x}\right)^{*} \times V$ into $M$ such that

$$
\left(\Psi_{\alpha} \circ \varphi\right)\left(a G_{x}, \eta, v\right)=\frac{\Psi_{\alpha}(x)+j(\eta)+i(\Phi(v))}{\left\|\Psi_{\alpha}(x)+j(\eta)+i(\Phi(v))\right\|} .
$$

Proof. Since the isotropy group $G_{x}$ is connected, the sequence $1 \rightarrow$ $G_{x} \rightarrow G \rightarrow G / G_{x} \rightarrow 1$ splits. Hence $G \times_{G_{x}}\left(\left(\mathfrak{k} / \mathfrak{g}_{x}\right)^{*} \times V\right)=G / G_{x} \times$ $\left(\mathfrak{k} / \mathfrak{g}_{x}\right)^{*} \times V$.

REMARK 3.15. Recall that for the standard representation of the $n$ torus $\mathbb{T}^{n}$ on $\mathbb{C}^{n}$ preserving the standard symplectic form $\omega=\sqrt{-1} \sum d z_{j} \wedge$ $d \bar{z}_{j}$, the corresponding moment map $\Phi: \mathbb{C}^{n} \rightarrow\left(\mathbb{R}^{n}\right)^{*}$ is given by $\Phi(z)=\left(\left|z_{1}\right|^{2}, \ldots,\left|z_{n}\right|^{2}\right)$. Hence the fibers of $\Phi$ are $\mathbb{T}^{n}$-orbits. Consequently if $\rho: H \rightarrow \operatorname{Sp}\left(V, \omega_{V}\right)$ is a faithful representation of a torus $H$ with $\operatorname{dim} V=2 \operatorname{dim} H$, then the fibers of the corresponding moment map $\Phi_{V}: V \rightarrow \mathfrak{h}^{*}$ are $H$-orbits.

Lemma 3.16. Let $\left(M, \alpha, \Psi_{\alpha}: M \rightarrow \mathfrak{g}^{*}\right)$ be a compact connected contact toric G-manifold. Then

1) The connected components of the fibers of $\Psi_{\alpha}$ are $G$-orbits.

2) For any point $x \in M$ and any sufficiently small G-invariant neighborhood $U$ of $x$ in $M$ the pair $\left(\mathbb{R}^{+} \Psi_{\alpha}(x), C\left(\left.\Psi_{\alpha}\right|_{U}\right)\right)$ determines the contact toric manifold $\left(U,\left.\alpha\right|_{U},\left.\Psi_{\alpha}\right|_{U}=\Psi_{\left.\alpha\right|_{U}}\right)$. (Recall that $\left.C\left(\left.\Psi_{\alpha}\right|_{U}\right)\right)=\left\{t \Psi_{\alpha}(x) \mid t \in[0, \infty), x \in U\right\}$ is the moment cone of $\left.\Psi_{\alpha}\right)$.

Proof. Fix a point $x \in M$. Let $\rho: G_{x} \rightarrow \operatorname{Sp}\left(V, \omega_{V}\right)$ denotes the corresponding symplectic slice representation and $\mathfrak{k}=\left(\mathbb{R} \Psi_{\alpha}(x)\right)^{\circ}$ the characteristic subalgebra. Let $i: \mathfrak{g}_{x}^{*} \rightarrow \mathfrak{g}^{*}$ and $j:\left(\mathfrak{k} / \mathfrak{g}_{x}\right)^{*} \rightarrow \mathfrak{g}^{*}$ be the embeddings as in Lemma 3.10.

By Lemma 3.13, the isotropy group $G_{x}$ is connected. By Lemma 3.10 there exists a $G$-invariant neighborhood $U$ of $G \cdot x$ in $M$, a $G$-invariant neighborhood $U_{0}$ of $G \cdot[1,0,0]$ in $N=G \times_{G_{x}}\left(\left(\mathfrak{k} / \mathfrak{g}_{x}\right)^{*} \times V\right)$, a $G$ invariant contact form $\alpha_{N}$ on $N$ and a $G$-equivariant diffeomorphism $\varphi: U_{0} \rightarrow U$ such that $\varphi^{*} \alpha=e^{f} \alpha_{N}$ for some $G$-invariant function $f$. Consequently the $\alpha$ - and $\alpha_{N}$-moment maps are related by $\Psi_{\alpha} \circ$ $\varphi([a, \eta, v])=e^{f([a, \eta, v])} \Psi_{\alpha_{N}}([a, \eta, v])$. Recall that the $\alpha_{N}$-moment map $\Psi_{\alpha_{N}}$ is given by $\Psi_{\alpha_{N}}([a, \eta, v])=\left(\Psi_{\alpha}(x)+j(\eta)+i\left(\Phi_{V}(v)\right)\right)$, where $\Phi_{V}: V \rightarrow \mathfrak{g}_{x}^{*}$ is the moment map for the slice representation.

As observed previously the connectedness of $G_{x}$ implies that $N$ is diffeomorphic to $G / G_{x} \times\left(\mathfrak{k} / \mathfrak{g}_{x}\right)^{*} \times V$. Under this identification $\Psi_{\alpha_{N}}\left(a G_{x}, \eta, v\right)=\Psi_{\alpha}(x)+j(\eta)+i\left(\Phi_{V}(v)\right)$. Hence a fiber of $\Psi_{\alpha_{N}}$ is 
of the form $G / G_{x} \times\{\eta\} \times \Phi_{V}^{-1}(\mu)$. Since the fibers of $\Phi_{V}$ are $G_{x}$-orbits (see Remark 3.15), the fibers of $\Psi_{\alpha_{N}}$ are $G$-orbits. It follows that for any point $\eta \in \mathfrak{g}^{*}$ the set $\Psi_{\alpha}^{-1}(\eta) \cap U$ is a $G$-orbit.

We next argue that the pair $\left(\mathbb{R}^{+} \Psi_{\alpha}(x), C\left(\left.\Psi_{\alpha}\right|_{U}\right)\right)$ determines $G_{x}$ and the symplectic slice representation $\rho$ (it obviously determines the characteristic subalgebra). It is no loss of generality to assume that $\left(M, \alpha, \Psi_{\alpha}\right)=\left(N, \alpha_{N}, \Psi_{\alpha_{N}}\right)$ (note that the contact form $\alpha_{N}$ is not normalized but this won't matter) and that $U$ is a neighborhood of $G / G_{x} \times\{0\} \times\{0\}$ in $N=G / G_{x} \times\left(\mathfrak{k} / \mathfrak{g}_{x}\right)^{*} \times V$. Since $G_{x}$ is connected it is determined by its Lie algebra $\mathfrak{g}_{x}$ or, equivalently, by its annihilator $\mathfrak{g}_{x}^{\circ}$. Let $C$ be the moment cone of $\left(U,\left.\alpha_{N}\right|_{U},\left.\Psi_{\alpha_{N}}\right|_{U}\right)$. We may assume that $U$ is of the form $G / G_{x} \times D_{1} \times D_{2}$ where $D_{1}$ is a neighborhood of 0 in $\left(\mathfrak{k} / \mathfrak{g}_{x}\right)^{*}$ and $D_{2}$ is a $G_{x^{-}}$-invariant neighborhood of 0 in $V$. Then $C=\{0\} \cup \mathbb{R}^{+}\left(\Psi_{\alpha}(x)+j\left(D_{1}\right)+i\left(\Phi_{V}\left(D_{2}\right)\right)\right)$. Note that $\mathbb{R}^{+}\left(\Psi_{\alpha}(x)+j\left(D_{1}\right)\right)$ is an open subset of $\mathfrak{g}_{x}^{\circ}$. Hence

$\mathfrak{g}_{x}^{\circ}=\left\{w \in \mathfrak{g}^{*} \mid\right.$ there is $\epsilon>0$ such that $\Psi_{\alpha}(x)+t w \in C$ for all $\left.t \in(-\epsilon, \epsilon)\right\}$.

Once we determined $\mathfrak{g}_{x}$ we have the natural inclusion $\iota: \mathfrak{g}_{x} \rightarrow \mathfrak{g}$ and the dual projection $\iota^{T}: \mathfrak{g}^{*} \rightarrow \mathfrak{g}_{x}^{*}$. Note that $\iota^{T} \circ i=i d_{\mathfrak{g}_{x}^{*}}$. Therefore $\iota^{T}(C)=\Phi_{V}(V)$. By Lemma 3.13, the cone $\Phi_{V}(V)$ completely determines the representation $\rho: G_{x} \rightarrow \operatorname{Sp}\left(V, \omega_{V}\right)$.

\section{Properties of contact moment maps}

In this section we gather a number of results describing the properties of contact moment maps, such as convexity of the image and connectedness of the fibers, and then apply them to moment maps on contact toric manifolds.

Definition 4.1. Let $\Psi_{\alpha}: M \rightarrow \mathfrak{g}^{*}$ be the moment map for an action of a torus $G$ on a manifold $M$ preserving a contact form $\alpha$. The corresponding orbital moment map is the map $\bar{\Psi}_{\alpha}: M / G \rightarrow \mathfrak{g}^{*}$ induced by $\Psi_{\alpha}$.

The first property is the convexity of the image and the connectedness of the fibers which is due to Banyaga and Molino in the toric case [BaM1, BaM2] (by Lemma 2.12 we know that Theorem 4.2 below applies to contact toric manifolds). ${ }^{3}$

\footnotetext{
${ }^{3}$ I don't understand the proof of convexity and connectedness in [BaM2]. In particular it is not clear to me how the hypothesis that the dimension of the group is at least 3 is being used.
} 
Theorem 4.2. Let $(M, \xi)$ be a co-oriented contact manifold with an effective action of a torus $G$ preserving the contact structure and its co-orientation. Let $\xi_{+}^{\circ}$ be a component of the annihilator of $\xi$ in $T^{*} M$ minus the zero section: $\xi^{\circ} \backslash 0=\xi_{+}^{\circ} \sqcup\left(-\xi_{+}^{\circ}\right)$. Assume that $M$ is compact and connected and that the dimension of $G$ is bigger than 2. If 0 is not in the image of the contact moment map $\Psi: \xi_{+}^{\circ} \rightarrow \mathfrak{g}^{*}$ then the fibers of $\Psi$ are connected and the moment cone $C(\Psi)=\Psi\left(\xi_{+}^{\circ}\right) \cup\{0\}$ is a convex rational polyhedral cone.

Proof. See $[\mathbf{L} \mathbf{1}]$.

Lemma 4.3. Let $\left(M, \alpha, \Psi_{\alpha}: M \rightarrow \mathfrak{g}^{*}\right)$ be a c.c.c.t. $G$-manifold normalized so that $\Psi_{\alpha}(M) \subset S\left(\mathfrak{g}^{*}\right)$. Suppose $\operatorname{dim} M>3$. Then the fibers of the moment map $\Psi_{\alpha}$ are $G$-orbits. Consequently the orbital moment map $\bar{\Psi}_{\alpha}: M / G \rightarrow S\left(\mathfrak{g}^{*}\right)$ is a (topological) embedding.

Proof. By Theorem 4.2, the fibers of $\Psi_{\alpha}$ are connected. By Lemma 3.16 the connected components of the fibers of $\Psi_{\alpha}$ are $G$-orbits. Therefore the fibers of $\Psi_{\alpha}$ are $G$-orbits and the orbital moment map is injective. Since $M / G$ is compact, the orbital moment map $\bar{\Psi}_{\alpha}$ is an embedding.

Lemma 4.3 does not hold for 3-dimensional contact toric manifolds. For example consider $(M, \alpha)=\left(S^{1} \times \mathbb{T}^{2}, \cos n t d \theta_{1}+\sin n t d \theta_{2}\right)$ with the corresponding moment map $\Psi_{\alpha}\left(t, \theta_{1}, \theta_{2}\right)=(\cos n t, \sin n t)$. The orbital moment map is $\bar{\Psi}_{\alpha}: S^{1}=M / \mathbb{T}^{2} \rightarrow S\left(\mathbb{R}^{2}\right)=S^{1}$ is given by $\bar{\Psi}_{\alpha}(t)=(\cos n t, \sin n t)$, which is not an embedding for $n>1$. However, $\bar{\Psi}_{\alpha}(t)$ is an embedding locally. This is true in general.

Lemma 4.4. Let $\left(M, \alpha, \Psi_{\alpha}: M \rightarrow \mathfrak{g}^{*}\right)$ be a c.c.c.t. $G$-manifold normalized so that $\Psi_{\alpha}(M) \subset S\left(\mathfrak{g}^{*}\right)$. For any $x \in M / G$ there is a neighborhood $U$ of $x$ in $M / G$ such that the restriction of the orbital moment map $\bar{\Psi}_{\alpha}$ to $U$ is an embedding into $S\left(\mathfrak{g}^{*}\right)$.

Proof. This is an easy consequence of the local normal form theorem, Theorem 3.14.

Lemma 4.5. Let $\left(M, \alpha, \Psi_{\alpha}: M \rightarrow \mathfrak{g}^{*}\right)$ be a c.c.c.t. $G$-manifold normalized so that $\Psi_{\alpha}(M) \subset S\left(\mathfrak{g}^{*}\right)$. Assume $\operatorname{dim} M>3$. If the moment map $\Psi_{\alpha}: M \rightarrow S\left(\mathfrak{g}^{*}\right)$ is onto, then the action of $G$ on $M$ is free, hence $\Psi_{\alpha}: M \rightarrow S\left(\mathfrak{g}^{*}\right)$ is a principal $G$-bundle.

Proof. Suppose the action of $G$ is not free. Then for some point $x \in M$ the isotropy group $G_{x}$ is not trivial. By Lemma 4.3 for any $G$-invariant 
neighborhood $U$ of the orbit $G \cdot x$ there is an open subset $\widetilde{W}$ of the sphere $S\left(\mathfrak{g}^{*}\right)$ such that

$$
\Psi_{\alpha}(U)=\widetilde{W} \cap \Psi_{\alpha}(M) .
$$

By the local normal form theorem, Theorem 3.14, we may choose $U$ and $\widetilde{W}$ so that

$$
\Psi_{\alpha}(U)=\widetilde{W} \cap\left\{\frac{\Psi_{\alpha}(x)+j(\eta)+i(\Phi(v))}{\left\|\Psi_{\alpha}(x)+j(\eta)+i(\Phi(v))\right\|} \mid \eta \in\left(\mathfrak{k} / \mathfrak{g}_{x}\right)^{*}, v \in V\right\}
$$

where as in Theorem $3.14 \rho: G_{x} \rightarrow \operatorname{Sp}\left(V, \omega_{V}\right)$ denotes the symplectic slice representation, $\Phi: V \rightarrow \mathfrak{g}^{*}$ denote the corresponding moment map, $\mathfrak{k}=\left(\mathbb{R} \Psi_{\alpha}(x)\right)^{\circ}$ is the characteristic subalgebra, and $i: \mathfrak{g}_{x}^{*} \rightarrow$ $\mathfrak{g}^{*}, j:\left(\mathfrak{k} / \mathfrak{g}_{x}\right)^{*} \rightarrow \mathfrak{g}^{*}$ are the embeddings as in Lemma 3.10. Since $G_{x}$ is nontrivial, the symplectic slice $V$ is not zero. It follows from Lemma 3.13 that $\Phi(V)$ is a proper cone in $\mathfrak{g}_{x}^{*}$. Therefore $\Psi_{\alpha} \cap \widetilde{W} \neq \widetilde{W}$, i.e., $\Psi_{\alpha}$ is not onto. Contradiction. Therefore the action of $G$ is free.

By Lemma 4.3, the fibers of $\Psi_{\alpha}$ are $G$-orbits. Therefore if the action of $G$ is free, then $\Psi_{\alpha}: M \rightarrow S\left(\mathfrak{g}^{*}\right)$ is a principal $G$-bundle.

The next lemma is a partial converse to Lemma 4.5.

Lemma 4.6. Suppose a Lie group $G$ acts on a manifold $M$ preserving a contact form $\alpha$. Let $\Psi_{\alpha}: M \rightarrow \mathfrak{g}^{*}$ denotes the corresponding moment map. Suppose the action of $G$ at a point $x$ is free and the value $\mu$ of moment map at $x$ is non-zero. Then $\pi_{\mu} \circ d\left(\Psi_{\alpha}\right)_{x}: T_{x} M \rightarrow \mathfrak{g}^{*} / \mathbb{R} \mu$ is onto. Here $\pi_{\mu}: \mathfrak{g}^{*} \rightarrow \mathfrak{g}^{*} / \mathbb{R} \mu$ is the obvious projection.

Proof. Since the action of $G$ at $x$ is free, for any $0 \neq X \in \mathfrak{g}$ the induced vector field $X_{M}$ is nonzero at $x: X_{M}(x) \neq 0$. Since the action of $G$ preserves $\alpha, 0=d \iota\left(X_{M}\right) \alpha+\iota\left(X_{M}\right) d \alpha$. Therefore, for any $v \in T_{x} M$

$$
\left\langle d\left(\Psi_{\alpha}\right)_{x}(v), X\right\rangle=d\left\langle\Psi_{\alpha}, X\right\rangle_{x}(v)=d \alpha_{x}\left(v, X_{M}(x)\right) .
$$

Now

$$
\begin{aligned}
\left(\mathfrak{g}^{*} / \mathbb{R} \mu\right)^{*}=\operatorname{ker} \mu & =\left\{X \in \mathfrak{g} \mid\left\langle\Psi_{\alpha}(x), X\right\rangle=0\right\} \\
& =\left\{X \in \mathfrak{g} \mid \alpha_{x}\left(X_{M}(x)\right)=0\right\} \\
& =\left\{X \in \mathfrak{g} \mid X_{M} \in \operatorname{ker} \alpha_{x}\right\} .
\end{aligned}
$$

Hence to prove that $\pi_{\mu} \circ d\left(\Psi_{\alpha}\right)_{x}: T_{x} M \rightarrow \mathfrak{g}^{*} / \mathbb{R} \mu$ is onto, it's enough to show that for any $0 \neq X \in \mathfrak{g}$ with $\alpha_{x}\left(X_{M}(x)\right)=0$ there is $v \in T_{x} M$ with $d \alpha_{x}\left(v, X_{M}(x)\right)=\left\langle d\left(\Psi_{\alpha}\right)_{x}(v), X\right\rangle \neq 0$. Since $\alpha$ is contact, $\left.d \alpha\right|_{\text {ker } \alpha}$ is nondegenerate. Therefore, for any $X_{M}(x) \neq 0$ with $\alpha_{x}\left(X_{M}(x)\right)=0$ there is $v \in \operatorname{ker} \alpha_{x}$ so that $d \alpha_{x}\left(v, X_{M}(x)\right) \neq 0$. 
Corollary 4.7. Let $\left(M, \alpha, \Psi_{\alpha}: M \rightarrow \mathfrak{g}^{*}\right)$ be a c.c.c.t. G-manifold normalized so that $\Psi_{\alpha}(M) \subset S\left(\mathfrak{g}^{*}\right)$. If the action of $G$ is free then the moment map $\Psi_{\alpha}: M \rightarrow S\left(\mathfrak{g}^{*}\right)$ is a submersion.

If additionally $\operatorname{dim} M>3$ then $\Psi_{\alpha}: M \rightarrow S\left(\mathfrak{g}^{*}\right)$ is a principal G-bundle.

Proof. By Lemma 4.6, the differential $d\left(\Psi_{\alpha}\right)_{x}: T_{x} M \rightarrow T_{\Psi_{\alpha}(x)} S\left(\mathfrak{g}^{*}\right)$ is surjective for all $x \in M$. Consequently the image $\Psi_{\alpha}(M)$ is open in the sphere. On the other hand the image is closed since $M$ is compact. Thus the image is the whole sphere and $\Psi_{\alpha}: M \rightarrow S\left(\mathfrak{g}^{*}\right)$ is a submersion. Since $M$ is compact it follows that $\Psi_{\alpha}: M \rightarrow S\left(\mathfrak{g}^{*}\right)$ is a fibration. If $\operatorname{dim} M>3$ then, by Lemma $4.3, \Psi_{\alpha}: M \rightarrow S\left(\mathfrak{g}^{*}\right)$ is a principal $G$-bundle.

Definition 4.8. Two contact toric $G$-manifolds $\left(M, \alpha, \Psi_{\alpha}\right)$ and $\left(M, \alpha^{\prime}, \Psi_{\alpha^{\prime}}\right)$ are locally isomorphic if

1) there exists a homeomorphism $\bar{\varphi}: M / G \rightarrow M^{\prime} / G$ and

2) for any point $x \in M / G$ there is a neighborhood $U \subset M / G$ containing it and an isomorphism of contact manifolds $\varphi_{U}: \pi^{-1}(U) \rightarrow$ $\left(\pi^{\prime}\right)^{-1}(\bar{\varphi}(U))$ such that $\pi^{\prime} \circ \varphi_{U}=\bar{\varphi} \circ \pi$ where $\pi: M \rightarrow M / G$ and $\pi^{\prime}: M \rightarrow M / G$ are the orbit maps (hence, by Remark 2.16, $\left.\varphi_{U}^{*} \alpha^{\prime}=\alpha\right)$.

Lemma 4.9. Let $\left(M_{1}, \alpha_{1}, \Psi_{\alpha_{1}}: M_{1} \rightarrow \mathfrak{g}^{*}\right)$ and $\left(M_{2}, \alpha_{2}, \Psi_{\alpha_{2}}: M_{2} \rightarrow\right.$ $\left.\mathfrak{g}^{*}\right)$ be two c.c.c.t. $G$-manifolds normalized so that $\Psi_{\alpha_{i}}\left(M_{i}\right) \subset S\left(\mathfrak{g}^{*}\right)$, $i=1,2$. Suppose there is a homeomorphism $\bar{\varphi}: M_{1} / G \rightarrow M_{2} / G$ so that $\bar{\Psi}_{\alpha_{2}} \circ \bar{\varphi}=\bar{\Psi}_{\alpha_{1}}$, where $\bar{\Psi}_{\alpha_{1}}, \bar{\Psi}_{\alpha_{2}}$ are orbital moment maps.

Then $\left(M_{1}, \alpha_{1}, \Psi_{\alpha_{1}}\right)$ and $\left(M_{2}, \alpha_{2}, \Psi_{\alpha_{2}}\right)$ are locally isomorphic.

Proof. Denote the orbit map $M_{i} \rightarrow M_{i} / G$ by $\pi_{i}, i=1,2$. We want to show that for any point $x \in M_{1}$ there is a $G$-invariant neighborhood $U_{1} \subset M_{1}$ and a $G$-equivariant diffeomorphism $\varphi_{U}: U_{1} \rightarrow U_{2}=$ $\pi_{2}^{-1}\left(\bar{\varphi}\left(\pi_{1}\left(U_{1}\right)\right)\right)$ such that $\varphi_{U}^{*} \alpha_{2}=\alpha_{1}$ and such that

$$
\pi_{2} \circ \varphi_{U}=\bar{\varphi} \circ\left(\left.\pi_{1}\right|_{U_{1}}\right) \text {. }
$$

Pick a point $x_{2} \in \pi_{2}^{-1}\left(\bar{\varphi}\left(x_{1}\right)\right)$. Then, since $\bar{\Psi}_{\alpha_{2}} \circ \bar{\varphi}=\bar{\Psi}_{\alpha_{1}}$, we have $\Psi_{\alpha_{2}}\left(x_{2}\right)=\Psi_{\alpha_{1}}\left(x_{1}\right)$. Also, given a $G$-invariant neighborhood $U_{1}$ of $x_{1}$ in $M_{1}$, let $U_{2}=\pi_{2}^{-1}\left(\bar{\varphi}\left(\pi_{1}\left(U_{1}\right)\right)\right)$. We have $\Psi_{\alpha_{2}}\left(U_{2}\right)=\bar{\Psi}_{\alpha_{2}}\left(\bar{\varphi}\left(\pi_{1}\left(U_{1}\right)\right)=\right.$ $\bar{\Psi}_{\alpha_{1}}\left(\pi_{1}\left(U_{1}\right)\right)=\Psi_{\alpha_{1}}\left(U_{1}\right)$. Therefore by Lemma 3.16 (2) if $U_{1}$ is sufficiently small there exists a $G$-equivariant contactomorphism $\varphi_{U}: U_{1} \rightarrow$ $U_{2}$. Hence, as remarked earlier, $\Psi_{\alpha_{2}} \circ \varphi_{U}=\Psi_{\alpha_{1}}$ and $\varphi_{U}^{*} \alpha_{2}=\alpha_{1}$. 
It remains to show that the map $\bar{\varphi}_{U}$ induced by $\varphi_{U}$ on $U_{1} / G$ is $\bar{\varphi}$. If $U_{1}$ is sufficiently small, then by Lemma 4.4 the maps $\bar{\Psi}_{\alpha_{i}}: U_{i} / G \rightarrow$ $S\left(\mathfrak{g}^{*}\right), i=1,2$, are embeddings. Since $\Psi_{\alpha_{2}} \circ \varphi_{U}=\Psi_{\alpha_{1}}, \bar{\Psi}_{\alpha_{2}} \circ \bar{\varphi}_{U}=\bar{\Psi}_{\alpha_{1}}$. Therefore $\bar{\varphi}_{U}=\left.\left(\left.\bar{\Psi}_{\alpha_{2}}\right|_{\Psi_{\alpha_{1}}\left(U_{1}\right)}\right)^{-1} \circ \bar{\Psi}_{\alpha_{1}}\right|_{\pi_{1}\left(U_{1}\right)}=\left.\bar{\varphi}\right|_{\pi_{1}\left(U_{1}\right)}$.

\section{From local to global}

In this section we prove that compact connected contact toric manifolds are classified by the elements of the first Čech cohomology of their orbit space with coefficients in a certain sheaf. The argument here is an adaptation of the argument in $[\mathbf{L T}]$ (which was due to Lerman, Tolman and Woodward), which, in tern, was an adaptation of the argument in [HS]. I recently learned that essentially the same idea was developed earlier by Boucetta and Molino [BoM].

Let $(M, \xi=\operatorname{ker} \alpha)$ be a co-oriented contact manifold. Recall that a vector field $\Xi$ is contact if its flow preserves the contact distribution $\xi$, or, equivalently, if $L_{\Xi} \alpha=f \alpha$ for some function $f \in C^{\infty}(M)$. A choice of a contact form $\alpha$ with ker $\alpha=\xi$ establishes a bijection between contact vector fields and functions: given a contact vector field $\Xi$ the corresponding function is $\alpha(\Xi)$. Conversely, given a function $f \in C^{\infty}(M)$ the corresponding contact vector field $\Xi_{f}$ is defined by

$$
\Xi_{f}=f Y_{\alpha}-\left(\left.d \alpha\right|_{\xi}\right)^{-1}\left(\left.d f\right|_{\xi}\right)
$$

where $Y_{\alpha}$ is the Reeb vector field of $\alpha$, that is, the unique vector field such that $\alpha\left(Y_{\alpha}\right)=1, \iota\left(Y_{\alpha}\right) d \alpha=0$. Note that since $\left.d \alpha\right|_{\xi}$ is nondegenerate, $\left(\left.d \alpha\right|_{\xi}\right)^{-1}\left(\left.d f\right|_{\xi}\right)$ is a well-defined vector field tangent to $\xi$. Also, if $\Xi$ is contact then

$$
L_{\Xi} \alpha=Y_{\alpha}(\alpha(\Xi)) \alpha .
$$

If a Lie group $G$ acts on the manifold $M$ and if the contact form $\alpha$ is $G$-invariant, then the form defines a bijection between $G$-invariant contact vector fields and $G$-invariant functions.

Lemma 5.1. Suppose $\left(M, \alpha, \Psi_{\alpha}: M \rightarrow \mathfrak{g}^{*}\right)$ is a contact toric $G$ manifold. For any G-invariant function $f$ the flow $\varphi_{t}^{f}$ of the corresponding contact vector field $\Xi_{f}$ preserves the contact form $\alpha$ and induces the identity map on the orbit space $M / G$. In particular $\Xi_{f}$ is tangent to G-orbits.

Proof. We first consider the special case of $f=1$. Then the corresponding contact vector field is the Reeb vector field $Y_{\alpha}$. By definition 
of $Y_{\alpha}$ the Lie derivative $L_{Y_{\alpha}} \alpha$ satisfies $L_{Y_{\alpha}} \alpha=d \iota\left(Y_{\alpha}\right) \alpha+\iota\left(Y_{\alpha}\right) d \alpha=$ $d(1)+0=0$. Hence for any $X \in \mathfrak{g}$

$$
L_{Y_{\alpha}}\left\langle\Psi_{\alpha}, X\right\rangle=L_{Y_{\alpha}}\left(\iota\left(X_{M}\right) \alpha\right)=\iota\left(L_{Y_{\alpha}} X_{M}\right) \alpha+\iota\left(X_{M}\right)\left(L_{Y_{\alpha}} \alpha\right) .
$$

Since $Y_{\alpha}$ is unique, it is $G$-invariant. Hence $L Y_{\alpha} X_{M}=-L_{X_{M}} Y_{\alpha}=0$ for any $X \in \mathfrak{g}$. And by the previous computation $L_{Y_{\alpha}} \alpha=0$. Therefore

$$
L_{Y_{\alpha}}=0+0 .
$$

Thus the Reeb vector field is tangent to the fibers of the moment map $\Psi_{\alpha}$. Since $\left(M, \alpha, \Psi_{\alpha}: M \rightarrow \mathfrak{g}^{*}\right)$ is toric, the connected components of the fibers of $\Psi_{\alpha}$ are $G$-orbits (see Lemma 3.16). Therefore the Reeb vector field is tangent to $G$-orbits. Hence for any $G$-invariant function $f, Y_{\alpha}(f)=0$ and consequently $L_{\Xi_{f}} \alpha=Y_{\alpha}(f) \alpha=0$. That is, the flow $\varphi_{t}^{f}$ of $\Xi_{f}$ preserves $\alpha$.

For any $X \in \mathfrak{g}$

$$
L_{\Xi_{f}}\left\langle\Psi_{\alpha}, X\right\rangle=L_{\Xi_{f}}\left(\iota\left(X_{M}\right) \alpha\right)=\iota\left(\left[\Xi_{f}, X_{M}\right]\right) \alpha+\iota\left(X_{M}\right)\left(L_{\Xi_{f}} \alpha\right)=0+0 .
$$

Since connected components of the fibers of $\Psi_{\alpha}$ are $G$-orbits, this proves that the contact vector field $\Xi_{f}$ is tangent to $G$-orbits for any invariant function $f$. Hence the flow of $\Xi_{f}$ induces the identity map on the orbit space $M / G$.

Proposition 5.2. For a fixed torus $G$, the isomorphism classes of contact toric G-manifold locally isomorphic to a given contact toric $G$-manifold $\left(M, \alpha, \Psi_{\alpha}\right)$ are in one-to-one correspondence with the elements of the first Čech cohomology group $H^{1}(M / G, \mathcal{S})$ where $\mathcal{S}$ is the sheaf of groups on the orbit space $M / G$ defined by

$$
\mathcal{S}(U)=\operatorname{Iso}\left(\pi^{-1}(U)\right)
$$

the group of isomorphisms of the contact toric manifold $\left(\pi^{-1}(U),\left.\alpha\right|_{\pi^{-1}(U)}\right.$, $\left.\Psi_{\alpha}\right|_{\pi^{-1}(U)}$ ) (cf. Definition 2.15). Here again $\pi: M \rightarrow M / G$ denotes the orbit map.

Proof. The argument is standard (compare [HS] Proposition 4.2, or [BoM]). Suppose $\left(M^{\prime}, \alpha^{\prime}, \Psi_{\alpha^{\prime}}\right)$ is a contact toric $G$-manifold locally isomorphic to $\left(M, \alpha, \Psi_{\alpha}\right)$. Fix a homeomorphism $\bar{\varphi}: \bar{M}=M / G \rightarrow$ $\bar{M}^{\prime}=M^{\prime} / G$. Choose an open cover $\left\{V_{i}\right\}$ of $\bar{M}$ such that for each $i$ there is a $G$-equivariant contact diffeomorphism $\sigma_{i}: \pi^{-1}\left(V_{i}\right) \rightarrow\left(\pi^{\prime}\right)^{-1}\left(\bar{\varphi}\left(V_{i}\right)\right)$ inducing $\bar{\varphi}$ on $V_{i}$ (here $\pi^{\prime}: M^{\prime} \rightarrow M^{\prime} / G$ is the orbit map). Let

$$
f_{i j}=\left.\sigma_{i} \circ \sigma_{j}^{-1}\right|_{V_{i} \cap V_{j}}
$$


it is a Čech 1-cocycle whose cohomology class in $H^{1}(\bar{M}, \mathcal{S})$ is independent of the choices made to define it.

Conversely, given an element of $H^{1}(\bar{M}, \mathcal{S})$ we can represent it by a Cech cocycle $\left\{f_{i j}: \pi^{-1}\left(V_{i} \cap V_{j}\right) \rightarrow \pi^{-1}\left(V_{i} \cap V_{j}\right)\right\}$. We construct the corresponding contact toric $G$-manifold by taking the disjoint union of the manifolds $\left(\pi^{-1}\left(V_{i}\right),\left.\alpha\right|_{\pi^{-1}\left(V_{i}\right)},\left.\Psi_{\alpha}\right|_{\pi^{-1}\left(V_{i}\right)}\right)$ and gluing $\pi^{-1}\left(V_{i}\right)$ to $\pi^{-1}\left(V_{j}\right)$ along $\pi^{-1}\left(V_{i} \cap V_{j}\right)$ using $f_{i j}$. The cocycle condition guarantees that the gluing is consistent.

Proposition 5.3. Let $\left(M, \alpha, \Psi_{\alpha}\right)$ be a contact toric $G$-manifold. Let $\pi: M \rightarrow M / G$ denote the orbit map, and let $\mathbb{Z}_{G}:=\operatorname{ker}\{\exp : \mathfrak{g} \rightarrow G\}$ denote the integral lattice of the torus $G$. There exists a short exact sequence of sheaves of groups

$$
0 \rightarrow \underline{\mathbb{Z}}_{G} \stackrel{j}{\rightarrow} \mathcal{C} \stackrel{\Lambda}{\rightarrow} \mathcal{S} \rightarrow 1
$$

where for a sufficiently small open subset $U$ of the orbit space $M / G$

1) $\mathbb{Z}_{G}(U):=C^{\infty}\left(\pi^{-1}(U), \mathbb{Z}_{G}\right)^{G}$

2) $\mathcal{C}(U)=C^{\infty}\left(\pi^{-1}(U)\right)^{G}$, the sheaf of "smooth" functions on $M / G$;

3) $\mathcal{S}(U):=\operatorname{Iso}\left(\pi^{-1}(U)\right)$ is the sheaf defined in Proposition 5.2.

Hence $\mathcal{S}$ is a sheaf of abelian groups and the cohomology groups $H^{i}(M / G, \mathcal{S})$ are defined for all indices $i \geq 0$.

Proof. Let $U$ be an open subset of $\bar{M}=M / G$ and let $f \in \mathcal{C}(U)$. By Lemma 5.1, the time $t$-flow $\varphi_{t}^{f}: \pi^{-1}(U) \rightarrow \pi^{-1}(U)$ induces the identity map on $U$ and preserves the contact form $\alpha$. Hence for any $t, \varphi_{t}^{f}$ is in $\mathcal{S}(U)$. We define the map $\Lambda: \mathcal{C}(U) \rightarrow \mathcal{S}(U)$ by

$$
\Lambda(f)=\varphi_{1}^{f}
$$

We next argue that $\Lambda$ is onto. Suppose $\varphi \in \mathcal{S}(U)$. By Theorem 3.1 of $[\mathbf{H S}]$, there exists a smooth $G$-invariant map $\sigma: \pi^{-1}(U) \rightarrow G$ such that

$$
\varphi(x)=\sigma(x) \cdot x
$$

for all $x \in \pi^{-1}(U)$. Moreover, if $U$ is contractible, then $\sigma(x)=$ $\exp (X(x))$ for some smooth $G$-invariant map $X: \pi^{-1}(U) \rightarrow \mathfrak{g}$. It's not hard to check that $x \mapsto \exp (X(x)) \cdot x$ is the time- 1 flow of the vector field $\tilde{X}(x):=\left(X(x)_{M}\right)(x)$. Note that $\tilde{X}$ is a $G$-invariant vector 
field: for any $g \in G$ and $x \in \pi^{-1}(U)$

$$
\begin{aligned}
\tilde{X}(g \cdot x)=(X(g \cdot x))_{M}(g \cdot x) & =\left.\frac{d}{d s}\right|_{s=0} \exp (s X(g \cdot x)) \cdot(g \cdot x) \\
& =\left.\frac{d}{d s}\right|_{s=0} g \cdot(\exp (s X(x)) \cdot x)=d g_{M}(\tilde{X}(x)),
\end{aligned}
$$

where the third equality holds because $X$ is $G$-invariant and $G$ is abelian.

We next prove that the Lie derivative of $\alpha$ with respect to $\tilde{X}$ is zero: $L_{\tilde{X}} \alpha=0$. To do this we recall a few facts about basic forms $[\mathbf{K}]$. Given an action of a compact Lie group $G$ on a manifold $M$, a form $\beta$ is basic if it is $G$-invariant and if for any $X \in \mathfrak{g}$, the contraction $\iota\left(X_{M}\right) \beta$ is zero (for zero forms we only require invariance). The set of basic forms is a subcomplex of the de Rham complex of differential forms, i.e., if $\beta$ is basic then so is $d \beta$. Also, if $\varphi: M \rightarrow M$ is a $G$-equivariant map inducing the identity on $M / G$ and $\beta$ is basic, then $\varphi^{*} \beta=\beta$. This is because it is a closed condition that holds on the open dense subset of points of principal orbit type.

We claim that $L_{\tilde{X}} \alpha$ is basic for the action of the torus $G$ on the manifold $\pi^{-1}(U)$. Note that $L_{\tilde{X}} \alpha=d \iota(\tilde{X}) \alpha+\iota(\tilde{X}) d \alpha$. Since $\tilde{X}$ and $\alpha$ are $G$-invariant, $\alpha(\tilde{X})$ is $G$-invariant hence basic. Therefore $d \iota(\tilde{X}) \alpha$ is basic. Also, since $\tilde{X}$ and $\alpha$ are $G$-invariant, the second term $\iota(\tilde{X}) d \alpha$ is $G$-invariant. It remains to show that for any $Y \in \mathfrak{g}, 0=\iota\left(Y_{M}\right)[\iota(\tilde{X}) d \alpha]$. Now for any $Y, Z \in \mathfrak{g}$

$$
d \alpha\left(Y_{M}, Z_{M}\right)=Y_{M}\left(\alpha\left(Z_{M}\right)\right)-Z_{M}\left(\alpha\left(Y_{M}\right)\right)-\alpha\left(\left[Y_{M}, Z_{M}\right]\right)=0-0-0,
$$

because $\alpha\left(Y_{M}\right), \alpha\left(Z_{M}\right)$ are $G$-invariant functions and because $\left[Y_{M}, Z_{M}\right]=$ $-([Y, Z])_{M}=0$ (since $G$ is abelian). Therefore for any $x \in \pi^{-1}(U)$

$$
\iota\left(Y_{M}\right)[\iota(\tilde{X}) d \alpha]=d \alpha_{x}\left(Y_{M}(x),(X(x))_{M}(x)\right)=0 .
$$

Next let $\tau_{t}$ denote the time- $t$ flow of the vector field $\tilde{X}$. Clearly $\tau_{t}$ is $G$-equivariant and induces the identity map on $U$. Thus, since $\left(L_{\tilde{X}} \alpha\right)$ is basic we have $\left(\tau_{t}\right)^{*}\left(L_{\tilde{X}} \alpha\right)=\left(L_{\tilde{X}} \alpha\right)$ for all $t$. We also know that $\tau_{1}=\varphi$ and that $\varphi^{*} \alpha=\alpha$. Therefore

$$
\begin{aligned}
0=\varphi^{*} \alpha-\alpha=\tau_{1}^{*} \alpha-\tau_{0}^{*} \alpha & =\int_{0}^{1} \frac{d}{d t}\left(\tau_{t}^{*} \alpha\right) d t \\
& =\int_{0}^{1} \tau_{t}^{*}\left(L_{\tilde{X}} \alpha\right) d t=\int_{0}^{1}\left(L_{\tilde{X}} \alpha\right) d t=\left(L_{\tilde{X}} \alpha\right) .
\end{aligned}
$$


We conclude that $\tilde{X}$ is a contact vector field. We define $f=\alpha(\tilde{X})$. Then the contact vector field of $f$ is $\tilde{X}$ and $\Lambda(f)=\varphi_{1}^{f}=\tau_{1}=\varphi$. This concludes the proof that $\Lambda: \mathcal{C} \rightarrow \mathcal{S}$ is onto.

We define the map $j: \mathbb{Z}_{G} \rightarrow \mathcal{C}$ by $j(X)=\left\langle\Psi_{\alpha}, X\right\rangle$. Thus it remains to show that for any sufficiently small set $U \subset M / G$ and any function $f \in \mathcal{C}(U)$ if $\varphi_{1}^{f}(x)=x$ for all $x \in \pi^{-1}(U)$ then

$$
f=\left\langle\Psi_{\alpha}, X\right\rangle
$$

for some $X \in \mathbb{Z}_{G}$. In fact it is enough to show that the above equation holds on the open dense subset $\pi^{-1}\left(U_{0}\right)$ of $\pi^{-1}(U)$ consisting of the points where the action of $G$ is free. Now the contact vector field $\Xi_{f}$ of $f$ is $G$-invariant and is tangent to $G$-orbits (Lemma 5.1). Therefore there exists on $\pi^{-1}\left(U_{0}\right)$ a $G$-invariant smooth map $X: \pi^{-1}\left(U_{0}\right) \rightarrow \mathfrak{g}$ such that

$$
\Xi_{f}(x)=(X(x))_{M}(x) .
$$

Now the time-1 flow of $\tilde{X}(x):=(X(x))_{M}(x)$ is $x \mapsto \exp (X(x)) \cdot x$. Thus if $\varphi_{1}^{f}(x)=x$, then $\exp (X(x)) \cdot x=x$ for all $x \in \pi^{-1}\left(U_{0}\right)$. Hence $\exp (X(x))=1$ and so $X(x) \in \mathbb{Z}_{G}$ for all $x \in \pi^{-1}\left(U_{0}\right)$. Therefore, since $\mathbb{Z}_{G}$ is discrete and $X$ is continuous and since we may take $\pi^{-1}\left(U_{0}\right)$ to be connected, $X(x)=X$ for some fixed vector $X \in \mathbb{Z}_{G}$. It follows, since $\pi^{-1}\left(U_{0}\right)$ is dense in $\pi^{-1}(U)$ that $\Xi_{f}(x)=X_{M}(x)$ for all $x \in \pi^{-1}(U)$. Consequently $f=\alpha\left(\Xi_{f}\right)=\alpha\left(X_{M}\right)=\left\langle\Psi_{\alpha}, X\right\rangle$.

Corollary 5.4. Under the hypotheses of the proposition above,

$$
H^{i}(M / G, \mathcal{S})=H^{i+1}\left(M / G, \underline{\mathbb{Z}}_{G}\right)
$$

for all $i>0$.

Proof. The sheaf $\mathcal{C}$ is a fine sheaf, so the long exact sequence in cohomology induced by (5.2) breaks up for $i>0$ into isomorphisms $H^{i}(M / G, \mathcal{S}) \simeq H^{i+1}\left(M / G, \underline{\mathbb{Z}}_{G}\right)$.

\section{Proof of the classification theorem}

In this section we finally put the results of sections 4 and 5 to work and prove the classification theorem.

6.1. Proof of Theorem 2.18 (1). Suppose $\left(M, \alpha, \Psi_{\alpha}\right)$ is a c.c.c.t. $G=\mathbb{T}^{2}$ manifold and suppose the action of $G$ is free. Then the orbit space $M / G$ is a 1-dimensional compact connected manifold without boundary, hence is a circle $S^{1}$. Moreover, since any principal $\mathbb{T}^{2}$-bundle over $S^{1}$ is trivial, $M$ is diffeomorphic to $S^{1} \times \mathbb{T}^{2}=\mathbb{T}^{3}$. It remains to 
show that the contact form $\alpha$ equals $\alpha_{n}=\cos n t d \theta_{1}+\sin n t d \theta_{2}$ for some positive integer $n$.

By Lemma 4.6 the orbital moment map $\bar{\Psi}_{\alpha}: S^{1}=M / G \rightarrow S\left(\mathfrak{g}^{*}\right)=$ $S^{1}$ is a submersion, hence is a covering map. Therefore there is a homeomorphism $\bar{\varphi}: M / G \rightarrow S^{1}$ such that $\bar{\Psi}_{\alpha_{n}} \circ \bar{\varphi}=\bar{\Psi}_{\alpha}$ where $\Psi_{\alpha_{n}}$ is the $\alpha_{n}$-moment map and $n$ is the number of sheets in the cover $\bar{\Psi}_{\alpha}: S^{1} \rightarrow S^{1}$. By Lemma $4.9\left(M, \alpha, \Psi_{\alpha}\right)$ is locally isomorphic to $\left(M, \alpha_{n}, \Psi_{\alpha_{n}}\right)$. It follows from Proposition 5.2, Corollary 5.4 and the fact that $H^{2}\left(S^{1}, \mathbb{Z}^{2}\right)=0$ that $\left(M, \alpha, \Psi_{\alpha}\right)$ is isomorphic to $\left(M, \alpha_{n}, \Psi_{\alpha_{n}}\right)$.

\subsection{Proof of Theorem 2.18 (2).}

Lemma 6.1. Let $\left(M, \alpha, \Psi_{\alpha}\right)$ be a c.c.c.t. $G=\mathbb{T}^{2}$ manifold normalized so that $\Psi_{\alpha}(M) \subset S\left(\mathfrak{g}^{*}\right)$ and suppose the action of $G$ is not free. Then

1) The orbit space $M / G$ is homeomorphic to the interval [0,1].

2) The orbital moment map $\bar{\Psi}_{\alpha}: M / G \rightarrow S\left(\mathfrak{g}^{*}\right)=S^{1}$ lifts to an embedding $\tilde{\Psi}_{\alpha}: M / G \rightarrow \mathbb{R}$ so that $\Pi \circ \tilde{\Psi}_{\alpha}=\bar{\Psi}_{\alpha}$ where $\Pi: \mathbb{R} \rightarrow S^{1}$ is the covering map $\Pi(t)=(\cos t, \sin t)$.

3) $\tilde{\Psi}_{\alpha}(M / G)=\left[t_{1}, t_{2}\right]$, and $\tan t_{1}, \tan t_{2}$ are rational numbers.

4) If $\left(M^{\prime}, \alpha^{\prime}, \Psi_{\alpha^{\prime}}\right)$ is another c.c.c.t. G-manifold with $\tilde{\Psi}_{\alpha^{\prime}}\left(M^{\prime} / G\right)=$ $\tilde{\Psi}_{\alpha}(M / G)$ then $\left(M^{\prime}, \alpha^{\prime}, \Psi_{\alpha^{\prime}}\right)$ is isomorphic to $\left(M, \alpha, \Psi_{\alpha}\right)$.

5) Given $t_{1}, t_{2} \in \mathbb{R}$ with $0 \leq t_{1}<2 \pi, t_{1}<t_{2}$ and $\tan t_{1}$, tan $t_{2}$ rational, there is a c.c.c.t. G-manifold $\left(M, \alpha, \Psi_{\alpha}\right)$ with $\widetilde{\Psi}_{\alpha}(M / G)=$ $\left[t_{1}, t_{2}\right]$.

Proof. Since $\left(M, \alpha, \Psi_{\alpha}\right)$ is contact toric, $\Psi_{\alpha}(x) \neq 0$ for any $x \in M$ (Lemma 2.12). Therefore the action of $G$ on $M$ has no fixed points. By Lemma 3.13 all the isotropy groups are connected. Therefore they are either trivial or circles. Suppose the isotropy group $G_{x}$ at $x$ is a circle. Then its Lie algebra $\mathfrak{g}_{x}$ equals the characteristic subalgebra $\mathfrak{k}$. Hence $\Psi_{\alpha}(x)$ is a multiple of a weight $\mu \in \mathbb{Z}_{G}^{*}$.

Also, the dimension of the symplectic slice $V$ at $x$ is 2 . Hence the symplectic slice representation is isomorphic to the standard action of $S^{1}$ on $\mathbb{C}: \lambda \cdot z=\lambda z$. Consequently, by the local normal form theorem, Theorem 3.14, a neighborhood of $x$ in $M$ is diffeomorphic to $S^{1} \times \mathbb{C}$, and a neighborhood of $G \cdot x$ in $M / G$ is homeomorphic to $\mathbb{C} / S^{1}=[0, \infty)$. We conclude that $M / G$ is a 1-dimensional $C^{0}$ manifold with boundary. Since $M$ is compact and connected and since $G=\mathbb{T}^{2}$ it follows that

1) $M / G$ is homeomorphic to $[0,1]$;

2) there are exactly two orbits $G \cdot x_{1}, G \cdot x_{2}$ which are diffeomorphic to $S^{1}$ 
3) For $i=1,2, \Psi_{\alpha}\left(x_{i}\right)=\frac{\mu_{i}}{\left\|\mu_{i}\right\|}$ where $\mu_{i}=d \chi_{i}$ and the character $\chi_{i}$ is the map $G \rightarrow G / G_{x_{i}}$.

Since $G=\mathbb{T}^{2}$ we may identify $\mathfrak{g}^{*}$ with $\mathbb{R}^{2}$ and the weight lattice $Z_{G}^{*}$ with $\mathbb{Z}^{2}$. Then for any weight $\mu$ of $G, \frac{\mu}{\|\mu\|}=(\cos t, \sin t)$ for some $t \in \mathbb{R}$ with $\tan t$ rational.

Since $M / G$ is homeomorphic to $[0,1]$, the orbital moment map $\bar{\Psi}_{\alpha}$ : $M / G \rightarrow S\left(\mathfrak{g}^{*}\right)=S^{1}$ lifts to a map $\tilde{\Psi}_{\alpha}: M / G \rightarrow \mathbb{R}$ such that $\Pi \circ \tilde{\Psi}_{\alpha}=$ $\bar{\Psi}_{\alpha}$. Note that $\bar{\Psi}_{\alpha}(M / G)$ is an interval with end points being the images of the exceptional orbits $G \cdot x_{1}, G \cdot x_{2}$. Hence we may assume that $\bar{\Psi}_{\alpha}(M / G)=\left[t_{1}, t_{2}\right], 0 \leq t_{1}<2 \pi$, and that $\tan t_{1}, \tan t_{2}$ are rational numbers.

Moreover since $\bar{\Psi}_{\alpha}$ is locally an embedding (Lemma 4.4), $\widetilde{\Psi}_{\alpha}$ is an embedding. Thus $\tilde{\Psi}_{\alpha}: M / G \rightarrow\left[t_{1}, t_{2}\right]$ is a homeomorphism.

Now suppose $\quad\left(M^{\prime}, \alpha^{\prime}, \Psi_{\alpha^{\prime}}\right) \quad$ is another c.c.c.t. $G$-manifold with $\tilde{\Psi}_{\alpha^{\prime}}\left(M^{\prime} / G\right)=\left[t_{1}, t_{2}\right]$. Then $\varphi:=\left(\tilde{\Psi}_{\alpha^{\prime}}\right)^{-1} \circ \tilde{\Psi}_{\alpha}: M / G \rightarrow M^{\prime} / G$ is a homeomorphism with $\bar{\Psi}_{\alpha^{\prime}} \circ \varphi=\bar{\Psi}_{\alpha}$. By Lemma $4.9\left(M, \alpha, \Psi_{\alpha}\right)$ and $\left(M^{\prime}, \alpha^{\prime}, \Psi_{\alpha^{\prime}}\right)$ are locally isomorphic. Since $M / G$ is contractible, $H^{2}\left(M / G, \mathbb{Z}^{2}\right)=0$. By Corollary $5.4 H^{1}(M / G, \mathcal{S})=H^{2}\left(M / G, \mathbb{Z}^{2}\right)$, where $\mathcal{S}$ is the sheaf in Proposition 5.2. Hence, by Proposition 5.2, $\left(M, \alpha, \Psi_{\alpha}\right)$ and $\left(M^{\prime}, \alpha^{\prime}, \Psi_{\alpha^{\prime}}\right)$ are isomorphic.

To prove part (5) we use an equivariant version of Proposition 2.15 in $[\mathbf{L 2}]$ :

Proposition 6.2. Suppose $(\tilde{M}, \alpha)$ is a contact manifold, $M$ is a manifold with boundary of the same dimension as $\tilde{M}$ embedded in $\tilde{M}$. Suppose further that there is a neighborhood $U$ in $\tilde{M}$ of the boundary $\partial M$ and a free $S^{1}$ action on $U$ preserving $\alpha$ such that the corresponding moment map $f: U \rightarrow \mathbb{R}$ satisfies

1) $f^{-1}(0)=\partial M$ and

2) $f^{-1}([0, \infty))=U \cap M$.

Let $M_{\text {cut }}=M / \sim$, where, for $m \neq m^{\prime}, m \sim m^{\prime}$ if and only if

1) $m, m^{\prime} \in \partial M$ and

2) $m=\lambda \cdot m^{\prime}$ for some $\lambda \in S^{1}$,

Then $M_{\text {cut }}$ is a contact manifold, $\partial M / S^{1}$ is a contact submanifold of $M_{\text {cut }}$, and $M_{\text {cut }} \backslash\left(\partial M / S^{1}\right)$ is contactomorphic to $M \backslash \partial M$.

Moreover if there is an action of a Lie group $G$ on $\tilde{M}$ preserving $M$, $\alpha$ and commuting with the action of $S^{1}$ on $U$, then there is an induced action of $G$ on $M_{\text {cut }}$ preserving the induced contact structure. 
Suppose we are given $t_{1}, t_{2} \in \mathbb{R}$ with $\tan t_{1}, \tan t_{2}$ rational, $0 \leq t_{1}<$ $2 \pi$ and $t_{1}<t_{2}$. For each $i$ there is a weight $\left(m_{i}, n_{i}\right) \in \mathbb{Z}^{2}$ such that $\left(\cos t_{i}, \sin t_{i}\right)$ lies on the ray through $\left(m_{i}, n_{i}\right)$.

Choose $\epsilon>0$ sufficiently small so that $f_{1}(t)=-n_{1} \cos t+m_{1} \sin t$ is non-negative on $\left[t_{1}, t_{1}+\epsilon\right)$ and $f_{2}(t)=n_{2} \cos t-m_{2} \sin t$ is non-negative on $\left(t_{2}-\epsilon, t_{2}\right]$ and $t_{1}+\epsilon<t_{2}-\epsilon$. Consider $\tilde{M}=\mathbb{R} \times S^{1} \times S^{1}$ with the contact form $\alpha=\cos t d \theta_{1}+\sin t d \theta_{2},\left(t, \theta_{1}, \theta_{2}\right) \in \mathbb{R} \times S^{1} \times S^{1}$. Let $M=\left[t_{1}, t_{2}\right] \times S^{1} \times S^{1}, U=\left(\left(t_{1}-\epsilon, t_{1}+\epsilon\right) \cup\left(t_{2}-\epsilon, t_{2}+\epsilon\right)\right) \times S^{1} \times S^{1}$. Consider $f: U \rightarrow \mathbb{R}$ given by $f\left(t, \theta_{1}, \theta_{2}\right)=-n_{1} \cos t+m_{1} \sin t$ for $t \in\left(t_{1}-\epsilon, t_{1}+\epsilon\right)$ and $f\left(t, \theta_{1}, \theta_{2}\right)=n_{2} \cos t+m_{2} \sin t$ for $t \in\left(t_{2}-\epsilon, t_{2}+\epsilon\right)$. The function $f$ is a moment map for a circle action on $U$ generated on $\left(t_{1}-\epsilon, t_{1}+\epsilon\right) \times S^{1} \times S^{1}$ by $-n_{1} \frac{\partial}{\partial \theta_{1}}+m_{1} \frac{\partial}{\partial \theta_{2}}$ and on $\left(t_{2}-\epsilon, t_{2}+\epsilon\right) \times S^{1} \times S^{1}$ by $n_{2} \frac{\partial}{\partial \theta_{1}}-m_{2} \frac{\partial}{\partial \theta_{2}}$.

Note that the obvious action of $G=S^{1} \times S^{1}$ on $\tilde{M}$ preserves $M, \alpha$, $U$ and commutes with the action of $S^{1}$ defined by $f$. Therefore we may apply Proposition 6.2 . The contact manifold $M_{\text {cut }}$ so obtained with the induced action of $G$ is the desired manifold $\left(M, \alpha, \Psi_{\alpha}\right)$.

REMARK 6.3. It is not hard to see that the manifolds $M_{\text {cut }}$ we constructed above out of $\mathbb{R} \times S^{1} \times S^{1}$ are lens spaces. Indeed, topologically they are obtained by from $\left[t_{1}, t_{2}\right] \times S^{1} \times S^{1}$ by dividing out the boundary components by circle actions, which is equivalent to gluing two solid tori along their boundaries by various automorphisms of the boundary.

6.3. Proof of Theorem 2.18 (3). Let $\left(M, \alpha, \Psi_{\alpha}\right)$ be a c.c.c.t. $G$ manifold normalized so that $\Psi_{\alpha}(M) \subset S\left(\mathfrak{g}^{*}\right)$, suppose the action of $G$ is free and suppose $\operatorname{dim} M>3$. By Corollary 4.7 the moment map $\Psi_{\alpha}: M \rightarrow S\left(\mathfrak{g}^{*}\right)$ is a principal $G$-bundle. By Lemma $4.9\left(M, \alpha, \Psi_{\alpha}\right)$ is locally isomorphic to the co-sphere bundle $S^{*} G=G \times S\left(\mathfrak{g}^{*}\right)$ of the torus $G$ with the standard contact structure and the obvious action of $G$. By Proposition $5.2\left(M, \alpha, \Psi_{\alpha}\right)$ corresponds to a class in $H^{1}(M / G, \mathcal{S})=H^{1}\left(S\left(\mathfrak{g}^{*}\right), \mathcal{S}\right)$. By Corollary $5.4 H^{1}\left(S\left(\mathfrak{g}^{*}\right), \mathcal{S}\right)$ is isomorphic to $H^{2}\left(S\left(\mathfrak{g}^{*}\right), \underline{\mathbb{Z}}_{G}\right)$. On the other hand the cohomology group $H^{2}\left(S\left(\mathfrak{g}^{*}\right), \underline{\mathbb{Z}}_{G}\right)$ classifies principal $G$-bundles over the sphere $S\left(\mathfrak{g}^{*}\right)$. This suggests (but doesn't yet prove!) that every principal $G$-bundle over $S\left(\mathfrak{g}^{*}\right)$ has a unique invariant contact structure. To prove this we need to trace through identifications.

Recall that principal $G$-bundles (for $G$ a torus) over $B=S\left(\mathfrak{g}^{*}\right)$ are in 1-1 correspondence with classes in the first Cech cohomology $H^{1}(B, \underline{G})$ where $\underline{G}$ is the sheaf defined by $\underline{G}(U)=C^{\infty}(U, G)$, for $U \subset B$ 
sufficiently small. Recall also that we have a short exact sequence of sheaves

$$
0 \rightarrow \underline{\mathbb{Z}}_{G} \rightarrow \underline{\mathfrak{g}} \stackrel{\exp }{\rightarrow} \underline{G} \rightarrow 1
$$

where $\underline{Z}_{G}(U)=C^{\infty}\left(U, \mathbb{Z}_{G}\right) \simeq \mathbb{Z}_{G}, \underline{\mathfrak{g}}(U)=C^{\infty}(U, \mathfrak{g})$ and $\exp : \underline{\mathfrak{g}} \rightarrow \underline{G}$ is induced by $\exp : \mathfrak{g} \rightarrow G$. On the other hand, by Proposition $\overline{5} .3$ we have the short exact sequence

$$
0 \rightarrow \underline{\mathbb{Z}}_{G} \stackrel{j}{\rightarrow} \mathcal{C} \stackrel{\Lambda}{\rightarrow} \mathcal{S} \rightarrow 1
$$

We claim that there are morphisms $a: \mathcal{C} \rightarrow \underline{\mathfrak{g}}$ and $b: \mathcal{S} \rightarrow \underline{G}$ so that the diagram

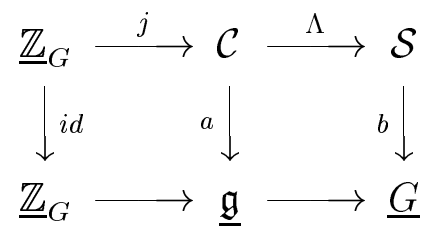

commutes and that, moreover, $b$ induces an isomorphism $H^{1}\left(S\left(\mathfrak{g}^{*}\right), \mathcal{S}\right) \rightarrow$ $H^{1}\left(S\left(\mathfrak{g}^{*}\right), \underline{G}\right)$.

Denote the projection $M \rightarrow S\left(\mathfrak{g}^{*}\right)$ by $\pi$. Fix an open set $U \subset S\left(\mathfrak{g}^{*}\right)$ small enough so that $\pi^{-1}(U)=U \times G$. By Lemma 5.1 for any $f \in \mathcal{C}(U)$ the contact vector field $\Xi_{f}$ of $f$ is tangent to $G$-orbits, i.e., to the fibers of $\pi$. Since the vector field $\Xi_{f}$ is also $G$-invariant, for any $x \in U$ there is a unique $X(x) \in \mathfrak{g}$ such that for any $m \in \pi^{-1}(x)$

$$
\Xi_{f}(m)=(X(x))_{M}(m)
$$

(cf. proof of Proposition 5.3). We define $a(f)$ to be the map $X: U \rightarrow \mathfrak{g}$, $x \mapsto X(x)$. Since an element $\varphi \in \mathcal{S}(U)$ is a $G$-equivariant diffeomorphism of $\pi^{-1}(U)=U \times G$ into itself, it is completely determined by $\left.\varphi\right|_{U \times\{1\}}$. We define $b: \mathcal{S}(U) \rightarrow \underline{G}$ by $b(\varphi)(x)=\varphi(x, 1)$.

By definition of $\Lambda: \mathcal{C} \rightarrow \mathcal{S}, \Lambda(f)$ is the time 1 flow of $\Xi_{f}$. Thus if $\Xi_{f}(m)=(X(\pi(m)))_{M}(m)$ then $\Lambda(f)(m)=(\exp X(\pi(m))) \cdot m=$ $\exp (a(f)(\pi(m))) \cdot m$. Consequently $b(\Lambda(f))(x)=\exp (a(f)(x))$, i.e., $b \circ \Lambda=\exp \circ a$.

Finally the left hand square commutes by definition of $j$ and the fact that the contact vector field of the function $\left\langle\Psi_{\alpha}, X\right\rangle$ is $X_{M}$. This proves the claim. 
Since $\mathcal{C}$ and $\underline{\mathfrak{g}}$ are fine sheaves we have

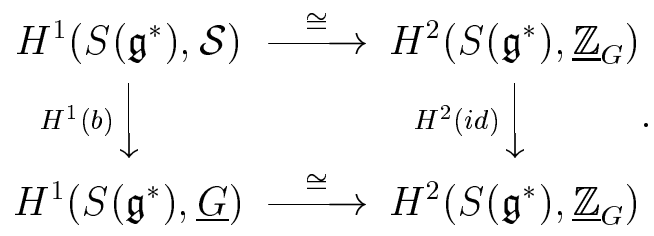

Therefore the map $H^{1}(b)$ induced by $b$ on the first Čhech cohomology is an isomorphism.

\subsection{Proof of Theorem 2.18 (4).}

Lemma 6.4. Let $\mathfrak{g}^{*}$ be the dual of the Lie algebra of a torus $G$. Let $C \subset \mathfrak{g}^{*}$ be a good polyhedral cone. There exists a c.c.c.t. G-manifold $\left(M, \alpha, \Psi_{\alpha}\right)$ such that the moment cone of $\Psi_{\alpha}$ is $C$.

Proof. Suppose $C=\bigcap\left\{\eta \in \mathfrak{g}^{*} \mid\left\langle\eta, v_{i}\right\rangle \geq 0\right\}$ is a good polyhedral cone defined by some subset $\left\{v_{i}\right\}_{i=1}^{N}$ of the integral lattice $\mathbb{Z}_{G}$.

As a first step we construct a symplectic cone $(S, \omega)^{4}$ with a symplectic action of $G$ commuting with dilations such that the image of the corresponding moment map $\Phi_{S}: S \rightarrow \mathfrak{g}^{*}$ is $C$. The construction is a slight adaptation of a well-known construction of Delzant (c.f. [D], $[\mathbf{L T}])$.

Let $\left\{e_{i}\right\}$ denote the standard basis of $\mathbb{R}^{N}$. Consider the map $\varpi$ : $\mathbb{R}^{N} \rightarrow \mathfrak{g}$ given by $\varpi\left(\sum a_{i} e_{i}\right)=\sum a_{i} v_{i}$. Since $\varpi\left(\mathbb{Z}^{N}\right) \subset \mathbb{Z}_{G}, \varpi$ induces a map $\bar{\varpi}: \mathbb{T}^{N}=\mathbb{R}^{N} / \mathbb{Z}^{N} \rightarrow \mathfrak{g} / \mathbb{Z}_{G}=G$. We write $[a]$ for the image of $a=\left(a_{1}, \ldots, a_{N}\right) \in \mathbb{R}^{N}$ in $\mathbb{T}^{N}$. Note that the kernel $T$ of $\bar{\varpi}$ is

$$
T=\left\{[a] \mid \sum a_{i} v_{i} \in \mathbb{Z}_{G}\right\} .
$$

It is a compact abelian subgroup of $\mathbb{T}^{N}$ with Lie algebra $\mathfrak{t}=$ ker $\varpi$. Note that $T$ need not be connected.

Consider the standard action of $\mathbb{T}^{N}$ on $\left(\mathbb{C}^{N}, \frac{\sqrt{-1}}{2 \pi} \sum d z_{j} \wedge d \bar{z}_{j}\right)$ :

$$
[a] \cdot\left(z_{1}, \ldots, z_{N}\right)=\left(e^{2 \pi i a_{1}} z_{1}, \ldots e^{2 \pi i a_{N}} z_{N}\right) .
$$

The corresponding symplectic moment map $\Phi: \mathbb{C}^{N} \rightarrow\left(\mathbb{R}^{N}\right)^{*}$ is given by $\Phi\left(z_{1}, \ldots, z_{N}\right)=\sum\left|z_{j}\right|^{2} e_{j}^{*}$ where $\left\{e_{j}^{*}\right\}$ is the basis dual to $\left\{e_{j}\right\}$. We claim that the symplectic quotient $S$ of $\mathbb{C}^{N} \backslash\{0\}$ by the induced action of $T$ is the desired manifold $S$, i.e., that

$$
S=\left(\Phi_{T}^{-1}(0) \backslash\{0\}\right) / T
$$

\footnotetext{
${ }^{4}$ Recall that a symplectic cone is a symplectic manifold $(S, \omega)$ with a free proper action $\left\{\rho_{t}\right\}_{t \in \mathbb{R}}$ of the real line such that $\rho_{t}^{*} \omega=e^{t} \omega$ for all $t \in \mathbb{R}$
} 
where $\Phi_{T}=j^{*} \circ \Phi$ and $j: \mathfrak{t} \hookrightarrow \mathbb{R}^{N}$ is the inclusion.

We claim first that $\Phi_{T}^{-1}(0)=\Phi^{-1}(\varpi(C))$. Indeed, since $0 \rightarrow \mathfrak{g}^{*} \stackrel{\varpi^{*}}{\rightarrow}$ $\left(\mathbb{R}^{N}\right)^{*} \stackrel{j^{*}}{\rightarrow} \mathfrak{t}^{*} \rightarrow 0$ is exact, $\left(j^{*}\right)^{-1}(0)=\varpi^{*}\left(\mathfrak{g}^{*}\right)$ and hence $\Phi_{T}^{-1}(0)=$ $\Phi^{-1}\left(\left(j^{*}\right)^{-1}(0) \cap \Phi\left(\mathbb{C}^{N}\right)\right)=\Phi^{-1}\left(\varpi^{*}\left(\mathfrak{g}^{*}\right) \cap \Phi\left(\mathbb{C}^{N}\right)\right)$. Now

$$
\begin{aligned}
\varpi^{*}\left(\mathfrak{g}^{*}\right) \cap \Phi\left(\mathbb{C}^{N}\right) & =\left\{\varpi^{*}(\eta) \mid \eta \in \mathfrak{g}^{*} \text { and }\left\langle\varpi^{*}(\eta), e_{i}\right\rangle \geq 0 \text { for all } i\right\} \\
& =\left\{\varpi^{*}(\eta) \mid \eta \in \mathfrak{g}^{*} \text { and } 0 \leq\left\langle\eta, \varpi\left(e_{i}\right)\right\rangle=\left\langle\eta, v_{i}\right\rangle \text { for all } i\right\} \\
& =\left\{\varpi^{*}(\eta) \mid \eta \in C\right\} .
\end{aligned}
$$

Thus $\Phi_{T}^{-1}(0)=\Phi^{-1}\left(\varpi^{*}(C)\right)$.

Next we claim that for any $0 \neq z=\left(z_{1}, \ldots, z_{N}\right) \in \Phi_{T}^{-1}(0)$ the isotropy group $T_{z}$ is trivial. It would then follow that $S=\left(\Phi_{T}^{-1}(0) \backslash\right.$ $\{0\}) / T$ is a smooth symplectic cone: the action of $\mathbb{R}$ on $S$ is induced by the action of $\mathbb{R}$ on $\mathbb{C}^{N}$ given by $t \cdot z=e^{t} z$. Now $T_{z}=T \cap\left(\mathbb{T}^{N}\right)_{z}=\{[a] \in$ $\left.\mathbb{R}^{N} / \mathbb{Z}^{N} \mid \sum a_{i} v_{i} \in \mathbb{Z}_{G}\right\} \cap\left\{[a] \in \mathbb{R}^{N} / \mathbb{Z}^{N} \mid a_{i} \in \mathbb{Z}\right.$ for all $i$ with $z_{i} \neq$ $0\}=\left\{[a] \in \mathbb{R}^{N} / \mathbb{Z}^{N} \mid \sum_{j \in J_{z}} a_{j} v_{j} \in \mathbb{Z}_{G}\right.$ and $a_{j} \in \mathbb{Z}$ for all $\left.j \notin J_{z}\right\}$ where $J_{z}=\left\{j \in\{1, \ldots, N\} \mid z_{j}=0\right\}$.

On the other hand, $z \in \Phi_{T}^{-1}(0)$ if and only if there is a (unique) $\eta \in C$ such that $\Phi(z)=\varpi^{*}(\eta)$. Hence $z_{j}=0$ if and only if $0=\left|z_{j}\right|^{2}=$ $\left\langle\Phi(z), e_{j}\right\rangle=\left\langle\varpi^{*}(\eta), e_{j}\right\rangle=\left\langle\eta, v_{j}\right\rangle$. Since $C$ is a good cone, for any fixed vector $\eta \in C$ the set $\left\{v_{j} \mid\left\langle\eta, v_{j}\right\rangle=0\right\}=\left\{v_{j} \mid j \in J_{z}\right\}$ is a $\mathbb{Z}$ basis of $\left\{\sum_{j \in J_{z}} a_{j} v_{j} \mid a_{j} \in \mathbb{R}\right\} \cap \mathbb{Z}_{G}$. Hence $\sum_{j \in J_{z}} a_{j} v_{j} \in \mathbb{Z}_{G}$ implies that $a_{j} \in \mathbb{Z}$ for all $j \in J_{z}$. Therefore $T_{z}=\left\{[a] \in \mathbb{R}^{N} / \mathbb{Z}^{N} \mid a \in \mathbb{Z}^{N}\right\}$, i.e., $T_{z}$ is trivial.

Finally note that the image of $\Phi_{T}^{-1}(0)$ under $\Phi$ is precisely $\varpi^{*}(C)$. Hence the image of the reduced space $S=\Phi_{T}^{-1}(0) / T$ under the induced $\mathbb{T}^{N} / T=G$ moment map $\tilde{\Phi}: \Phi_{T}^{-1}(0) / T \rightarrow \mathfrak{t}^{\circ}=\varpi^{*}\left(\mathfrak{g}^{*}\right)$ is $\varpi^{*}(C) \simeq C$.

Since the sphere $S^{2 N-1}=\left\{z \in \mathbb{C}^{N} \mid\|z\|^{2}=1\right\}$ is a $\mathbb{T}^{N}$-invariant hypersurface of contact type in $\mathbb{C}^{N}$, and since the action of $\mathbb{R}$ on $\mathbb{C}^{N}$ commutes with the action of $\mathbb{T}^{N}$

$$
M:=\left(\Phi_{T}^{-1}(0) \cap S^{2 N-1}\right) / T
$$

is a $\mathbb{T}^{N} / T=G$-invariant hypersurface of contact type in the quotient $\Phi_{T}^{-1}(0) / T$. Moreover $\left.\tilde{\Phi}\right|_{M}: M \rightarrow \mathfrak{t}^{\circ}=\varpi^{*}\left(\mathfrak{g}^{*}\right)$ is the corresponding contact moment map, and its moment cone is precisely $\varpi^{*}(C) \simeq C$.

Lemma 6.5. Suppose $\left(M, \alpha, \Psi_{\alpha}: M \rightarrow \mathfrak{g}^{*}\right)$ is a c.c.c.t. G-manifold, $\operatorname{dim} M>3$ and the action of $G$ is not free. Then the moment cone $C(\Psi)$ is a good rational polyhedral cone. 
Proof. We first introduce some notation and a simple fact. Let $C \subset \mathfrak{g}^{*}$ be a cone and $F \subset C$ be a face of $C$. Let $\operatorname{span}_{\mathbb{R}} F$ denote the linear subspace of $\mathfrak{g}^{*}$ spanned by the vectors in $F$. Let $\pi_{F}: \mathfrak{g}^{*} \rightarrow \mathfrak{g}^{*} / \operatorname{span}_{\mathbb{R}} F$ denote the projection. For any point $q$ in the interior of $F$ there is an open neighborhood $\mathcal{W}$ of $q$ in $\mathfrak{g}^{*}$ such that

$$
C \cap \mathcal{W}=\pi_{F}^{-1}\left(\pi_{F}(C)\right) \cap \mathcal{W} .
$$

Note that the cone $\pi_{F}^{-1}\left(\pi_{F}(C)\right)$ is isomorphic to $\pi_{F}(C) \times \operatorname{span}_{\mathbb{R}} F$.

Now suppose that $C$ is the moment cone $C(\Psi)$ of $\Psi_{\alpha}$ and that $x$ is a point in $M$. By Lemma 4.3 for any $G$ invariant neighborhood $U$ of $G \cdot x$ there is an open subset $\tilde{W}$ of the sphere $S\left(\mathfrak{g}^{*}\right)$ such that $\Psi_{\alpha}(U)=\tilde{W} \cap \Psi_{\alpha}(M)$. Let $W=\mathbb{R}^{+} \tilde{W} \cup\{0\}$ be the cone on $\tilde{W}$. Then

$$
C\left(\left.\Psi_{\alpha}\right|_{U}\right)=C\left(\Psi_{\alpha}\right) \cap W .
$$

By the local normal form theorem, Theorem 3.14,

$$
C\left(\left.\Psi_{\alpha}\right|_{U}\right)=W \cap\left(\mathbb{R}^{+}\left(\Psi_{\alpha}(x)+j\left(\left(\mathfrak{k} / \mathfrak{g}_{x}\right)^{*}\right)+i\left(\Phi_{V}(V)\right)\right) \cup\{0\}\right),
$$

where as usual $\mathfrak{k}$ is the characteristic subalgebra, $\Phi_{V}: V \rightarrow \mathfrak{g}_{x}^{*}$ is the moment map for the slice representation etc.. Note that

$$
W \cap \mathbb{R}^{+}\left(\Psi_{\alpha}(x)+j\left(\left(\mathfrak{k} / \mathfrak{g}_{x}\right)^{*}\right)\right)=W \cap \mathfrak{g}_{x}^{\circ}
$$

for any sufficiently "small" open cone $W$. Thus

$$
C\left(\left.\Psi_{\alpha}\right|_{U}\right)=W \cap\left(\mathfrak{g}_{x}^{\circ}+i\left(\Phi_{V}(V)\right)\right)=W \cap \pi_{x}^{-1}\left(\Phi_{V}(V)\right),
$$

where $\pi_{x}: \mathfrak{g}^{*} \rightarrow \mathfrak{g}_{x}^{*}$ is the natural projection. It follows that if $F$ is the face of $C\left(\Psi_{\alpha}\right)$ containing $\Psi_{\alpha}(x)$ in its interior, then $\operatorname{span}_{\mathbb{R}} F=\mathfrak{g}_{x}^{\circ}$ and $\pi_{F}\left(C\left(\Psi_{\alpha}\right)\right.$ ) is isomorphic to $\Phi_{V}(V)$ (once we identify $\mathfrak{g}^{*} / \mathfrak{g}_{x}^{\circ}$ with $\mathfrak{g}_{x}^{*}$ ).

Moreover, if we represent $C$ as $C=\bigcap\left\{\eta \in \mathfrak{g}^{*} \mid\left\langle\eta, v_{i}\right\rangle \geq 0\right\}$ for some minimal set $\left\{v_{i}\right\} \subset \mathbb{Z}_{G}$ and consisting of primitive vectors, then $\mathfrak{g}_{x}^{\circ}=$ $\operatorname{span}_{\mathbb{R}} F=\bigcap_{j=1}^{k}\left\{\eta \in \mathfrak{g}^{*} \mid\left\langle\eta, v_{i_{j}}\right\rangle=0\right\}$ for some subset $\left\{v_{i_{1}}, \ldots, v_{i_{k}}\right\} \subset$ $\mathbb{Z}_{G}$, and

$$
W \cap C\left(\Psi_{\alpha}\right)=W \cap\left(\pi_{x}^{-1}\left(\Phi_{V}(V)\right)\right)=W \cap \bigcap_{j=1}^{k}\left\{\eta \in \mathfrak{g}^{*} \mid\left\langle\eta, v_{i_{j}}\right\rangle \geq 0\right\} .
$$

Hence $\pi_{x}^{-1}\left(\Phi_{V}(V)\right)=\bigcap_{j=1}^{k}\left\{\eta \in \mathfrak{g}^{*} \mid\left\langle\eta, v_{i_{j}}\right\rangle \geq 0\right\}$. Since $\mathbb{Z}_{G_{x}}=\mathfrak{g}_{x} \cap \mathbb{Z}_{G}$, $\left\{v_{i_{j}}\right\}$ is a subset of $\left(\mathfrak{g}_{x}^{\circ}\right)^{\circ} \cap \mathbb{Z}_{G}=\mathfrak{g}_{x} \cap \mathbb{Z}_{G}=\mathbb{Z}_{G_{x}}$. For any $v \in \mathfrak{g}_{x}$ we have $\left.\pi_{x}\left(\left\{\eta \in \mathfrak{g}^{*} \mid\langle\eta, v\rangle \geq 0\right\}\right)=\left\{\eta \in \mathfrak{g}_{x}^{*} \mid\langle\eta, v\rangle \geq 0\right\}\right)$. Therefore

$$
\Phi_{V}(V)=\pi_{x}\left(\bigcap_{j=1}^{k}\left\{\eta \in \mathfrak{g}^{*} \mid\left\langle\eta, v_{i_{j}}\right\rangle \geq 0\right\}\right)=\bigcap_{j=1}^{k}\left\{\eta \in \mathfrak{g}_{x}^{*} \mid\left\langle\eta, v_{i_{j}}\right\rangle \geq 0\right\}
$$


By minimality of $\left\{v_{i}\right\}$ the set $\left\{v_{i_{j}}\right\}$ is the minimal set with this property. On the other hand, by Lemma 3.13,

$$
\Phi_{V}(V)=\left\{\sum_{j=1}^{k} a_{j} \nu_{j} \mid a_{j} \geq 0\right\}
$$

for some basis $\left\{\nu_{j}\right\}$ of $\mathbb{Z}_{G_{x}}^{*}$. Therefore the set $\left\{v_{i_{j}}\right\}$ is a basis of the lattice $\mathbb{Z}_{G_{x}}$.

\section{Uniqueness of toric integrable actions on the punctured cotangent bundles of $\mathbb{T}^{n}$ and $S^{2}$}

Having proved the classification theorem, Theorem 2.18, we are now in position to prove two applications: Theorems 1.3 and 1.5.

Proof of Theorem 1.3. Let $\left(M, \alpha, \Psi_{\alpha}: M \rightarrow \mathfrak{g}^{*}\right)$ be a contact toric $G$-manifold $\left(G=\mathbb{T}^{n}\right)$ such that $(M, \alpha)$ is contactomorphic to the co-sphere bundle $S^{*} \mathbb{T}^{n}$ of the $n$-torus $\mathbb{T}^{n}$ with the standard contact structure. We will argue that $\left(M, \alpha, \Psi_{\alpha}\right)$ is unique as a contact toric manifold.

It was shown in $[\mathbf{L S}]$ that if $M=S^{*} \mathbb{T}^{n}$, then the action of $G$ is necessarily free. The argument roughly goes as follows (see $[\mathbf{L S}]$ for details). Suppose the action of $G$ is not free. Consider first the case of $\operatorname{dim} M=3$. Then $M$ is a lens space (cf. Theorem $2.18(2)$ ), hence cannot be $S^{*} \mathbb{T}^{2}=\mathbb{T}^{3}$.

Next consider the case of $\operatorname{dim} M>3$. Then the moment cone $C$ of $\left(M, \alpha, \Psi_{\alpha}\right)$ is a good polyhedral cone determining $\left(M, \alpha, \Psi_{\alpha}\right)$ uniquely (cf. Theorem $2.18(4)$ ). If the maximal linear subspace of $C$ has dimension $k>0$ then $C$ is isomorphic to the moment cone of $M^{\prime}=\mathbb{T}^{k} \times S^{2 n-1-k}$, where $M^{\prime}$ gets its contact toric structure as a hypersurface of contact type $\left\{(q, p, z) \in \mathbb{T}^{k} \times\left(\mathbb{R}^{k}\right)^{*} \times \mathbb{C}^{n-k}\right.$ | $\left.\|p\|^{2}+\|z\|^{4}=1\right\}$ in $T^{*} \mathbb{T}^{k} \times \mathbb{C}^{n-k}$. Consequently $M$ is homeomorphic to $M^{\prime}=\mathbb{T}^{k} \times S^{2 n-1-k} \neq \mathbb{T}^{n} \times S^{n-1}=S^{*} \mathbb{T}^{n}$.

Finally if the dimension of the maximal linear subspace of $C$ is zero, i.e., if $C$ is a proper cone, then by a theorem of Boyer and Galicki [BG] $M$ has a locally free circle action so that the quotient $M / S^{1}$ is a compact connected symplectic toric orbifold. The real odd-dimensional cohomology of a compact symplectic toric orbifold is zero. Consequently $\operatorname{dim} H^{1}(M, \mathbb{R}) \leq 1$. Hence $M \neq S^{*} T^{n}, n>1$.

We conclude that if $\left(M, \alpha, \Psi_{\alpha}: M \rightarrow \mathfrak{g}^{*}\right)$ is a contact toric $G$ manifold $\left(G=\mathbb{T}^{n}\right)$ and $M=S^{*} \mathbb{T}^{n}$ then the action of $G$ is necessarily free. We argue next that it is unique. 
Suppose $\operatorname{dim} M=3$ and the action of $G=\mathbb{T}^{2}$ is free. By the classification theorem $(M, \alpha)=\left(\mathbb{T}^{3}, \alpha_{k}=\cos k t d \theta_{1}+\sin k t d \theta_{2}\right), k=1,2, \ldots$ By a theorem of Giroux $[\mathbf{G i}],\left(\mathbb{T}^{3}, \alpha_{k}\right)$ and $\left(\mathbb{T}^{3}, \alpha_{l}\right)$ are distinct as contact manifolds for $k \neq l$. Since $\alpha_{1}$ is the standard contact structure on $S^{*} \mathbb{T}^{2}$, it follows that there is only one contact toric manifold contactomorphic to $\left(S^{*} \mathbb{T}^{2}, \alpha_{1}\right)$. In other words there is only one $\mathbb{T}^{2}$-action on $S^{*} \mathbb{T}^{2}$ making it a contact toric manifold.

Suppose next that $\operatorname{dim} M>3$ and the action of $G$ is free. By Theorem 2.18 (4), $M$ is a principal $G$-bundle over the sphere $S^{n-1}, n=$ $\operatorname{dim} G$, and each such principal $G$-bundle carries only one $G$-invariant contact structure. Now, principal $\mathbb{T}^{n}$ bundles over $S^{n-1}$ are in one-toone correspondence with elements of $H^{2}\left(S^{n-1}, \mathbb{Z}^{n}\right)$ which is 0 unless $n-1=2$, in which case it's $\mathbb{Z}^{3}$. Note however that no nontrivial $\mathbb{T}^{3}$ bundle over $S^{2}$ is homeomorphic to $S^{2} \times \mathbb{T}^{3}$. We conclude that if $\left(M, \alpha, \Psi_{\alpha}\right)$ is a contact toric $G$-manifold such that the action of $G$ is free, $M=S^{*} G$ and $\operatorname{dim} M>3$ then $\left(M, \alpha, \Psi_{\alpha}\right)$ is a unique contact toric $G$-manifold with such properties. In other words there is only one $\mathbb{T}^{n}$-action on $S^{*} \mathbb{T}^{n}$ making it a contact toric manifold.

Proof of Theorem 1.5. Suppose $\tau_{1}, \tau_{2}$ are two effective actions of the torus $G=\mathbb{T}^{2}$ on $M=S^{*} S^{2}=\mathbb{R} P^{3}$ preserving the standard contact structure. Let $\Psi_{1}, \Psi_{2}: M \rightarrow S^{1} \subset \mathfrak{g}^{*}$ be the moment maps for the actions corresponding to a normalized contact form $\alpha_{0}$ defining the standard contact structure. We will argue that the images $\Psi_{i}(M)$ are arcs in $S^{1}$ of length less than $\pi$ (hence $\Psi_{i}$ are one-to-one). Moreover we'll show that there is an element $A \in \mathrm{SL}\left(\mathfrak{g}^{*}\right)$ preserving the weight lattice $\mathbb{Z}_{G}^{*}$ such that $A\left(\Psi_{1}(M)\right)=\Psi_{2}(M)$. It would then follow that the action $\tau_{1}$ composed with the isomorphism of $G$ defined by $A$ is $\tau_{2}$.

Note that since $\mathbb{R} P^{3} \neq \mathbb{T}^{3}$, the actions $\tau_{i}$ are necessarily not free (cf. Theorem 2.18 and the proof of Theorem 1.3 above). Now consider one of the two actions, say $\tau_{1}$. By Lemma 6.1 the action is free except at two orbits $G \cdot x_{1}$ and $G \cdot x_{2}$. The isotropy groups $K_{i}$ of $x_{i}$ are circles, and the images $\Psi_{1}\left(x_{i}\right)$ are of the form $\frac{\mu_{i}}{\left\|\mu_{i}\right\|}$ where $\mu_{i} \in \mathbb{Z}_{G}^{*}$ are primitive weights such that ker $\mu_{i}$ is the Lie algebra of the circle $K_{i}$.

It follows from the proof of Lemma 6.1 that the contact toric manifold $\left(M, \alpha_{0}, \Psi_{1}: M \rightarrow \mathfrak{g}^{*}\right)$ can be obtained by cutting $(\tilde{B}, \tilde{\alpha})=\left(\mathbb{T}^{2} \times\right.$ $\left.\mathbb{R}, \cos t d \theta_{1}+\sin t d \theta_{2}\right)$ using $B=\mathbb{T}^{2} \times\left[t_{1}, t_{2}\right]$, where $\left(\cos t_{i}, \sin t_{i}\right)=\frac{\mu_{i}}{\left\|\mu_{i}\right\|}$ (we identify $\mathfrak{g}^{*}$ with $\mathbb{R}^{2}$ and $\mathbb{Z}_{G}^{*}$ with $\mathbb{Z}^{2}$ ). Hence as a topological space $M=B / \sim$ where $\left(g, t_{1}\right) \sim\left(a g, t_{1}\right)$ for all $a \in K_{1}$ and $\left(g, t_{2}\right) \sim\left(a g, t_{2}\right)$ 
for all $a \in K_{2}$. Note that $t_{2}-t_{1} \neq \pi n, n=1,2, \ldots$, for otherwise $\mu_{1}= \pm \mu_{2}$ and then $B / \sim$ is $S^{2} \times S^{1} \neq \mathbb{R} P^{3}$.

Next observe that since the standard contact structure on $S^{*} S^{2}=M$ is tight, we must have $t_{2}-t_{1}<\pi$ (cf. [L2]). Indeed, if $t_{1}=0$ and $t_{2}>\pi$, the image of the cylinder $\left\{\left(1, e^{i \theta}, t\right) \mid 0 \leq t \leq t_{2}, \theta \in \mathbb{R}\right\} \subset B \subset \mathbb{T}^{2} \times \mathbb{R}$ in $M=B / \sim$ is an overtwisted disk. One can write a similar formula for an overtwisted disk if $t_{1}>0$. We conclude that the image $\Psi_{1}(M)$ in $S^{1}$ is an arc of length less than $\pi$. Consequently the fibers of $\Psi_{1}$ : $M \rightarrow S^{1}$ are connected.

We next argue that the weights $\mu_{1}, \mu_{2}$, which span the edges of the moment cone $C\left(\Psi_{1}\right)$, span a sublattice of the weight lattice $\mathbb{Z}_{G}^{*}$ of index two.

Lemma 7.1. Let $G=\mathbb{T}^{2}$ and let $K_{1}, K_{2} \subset G$ be two closed subgroups isomorphic to $S^{1}$. Let $M$ be the topological space $(G \times[0,1] / \sim$ where $(0, g) \sim(0, a g)$ for all $g \in G, a \in K_{1}$ and $(1, g) \sim(1, a g)$ for all $g \in G$ and $a \in K_{2}$. In other words $M$ is obtained from the manifold with boundary $G \times[0,1]$ by collapsing circles in the two components of the boundary by the respective actions of two circle subgroups. Let $\mu_{1}, \mu_{2} \in Z_{G}^{*}$ be the two primitive weights determined by $K_{1}$ and $K_{2}$ respectively, i.e., the kernel of the character defined by $\mu_{i}$ is $K_{i}$.

Then $H^{1}(M, \mathbb{Z}) \simeq\left\{\left(n_{1}, n_{2}\right) \in \mathbb{Z}^{2} \mid n_{1} \mu_{1}+n_{2} \mu_{2}=0\right\}$ and $H^{2}(M, \mathbb{Z}) \simeq$ $\mathbb{Z}_{G}^{*} /\left(\mathbb{Z} \mu_{1}+\mathbb{Z} \mu_{2}\right)$.

Proof. Recall that $H^{1}(G, \mathbb{Z})$ is isomorphic to the weight lattice $\mathbb{Z}_{G}^{*}$ and that the isomorphism is given as follows. A weight $\nu \in \mathbb{Z}_{G}^{*}$ defines a character $\chi_{\nu}: G \rightarrow S^{1}$ by $\chi_{\nu}(\exp (X))=e^{2 \pi i \nu(X)}$; the class $\chi_{\nu}^{*}[d \theta]$ is the element in $H^{1}(G, \mathbb{Z})$ corresponding to $\nu$. Here $d \theta$ is the obvious 1-form on $S^{1}$.

Consequently if $G=\mathbb{T}^{2}$ and $K_{j} \subset G$ is a circle subgroup, then $\pi_{j}: G \rightarrow G / K_{j} \simeq S^{1}$ is a character and hence the weight $\mu_{j}=\left(d \pi_{j}\right)_{1}$ defines an element of $H^{1}(G, \mathbb{Z})$. Thus if we identify $H^{1}\left(G / K_{j}, \mathbb{Z}\right)$ with $\mathbb{Z}$ and $H^{1}(G, \mathbb{Z})$ with $\mathbb{Z}_{G}^{*}$, then the $\operatorname{map} H^{1}\left(G / K_{j}, \mathbb{Z}\right) \rightarrow H^{1}(G, \mathbb{Z})$ becomes the map $\mathbb{Z} \ni n \mapsto n \mu_{j} \in \mathbb{Z}_{G}^{*}$.

The sets $U=(G \times[0,2 / 3)) / \sim$ and $V=(G \times(1 / 3,1]) / \sim$ are two open subsets of $M$. We have $M=U \cup V, U \cap V=G \times(1 / 3,2 / 3)$ is homotopy equivalent to $G, U$ is homotopy equivalent to $G / K_{1}, V$ is homotopy equivalent to $G / K_{2}$ and the inclusion maps $U \cap V \hookrightarrow U$, $U \cap V \hookrightarrow V$ are homotopy equivalent to projections $\pi_{1}: G \rightarrow G / K_{1}$, $\pi_{2}: G \rightarrow G / K_{2}$ respectively. Hence under the above identifications of 
$H^{1}(U)$ and $H^{1}(V)$ with $\mathbb{Z}$, the inclusions $U \cap V \rightarrow U, U \cap V \rightarrow V$ induce the maps $\mathbb{Z} \ni n \mapsto n \mu_{j} \in \mathbb{Z}_{G}^{*}, j=1,2$, respectively.

We now apply the Mayer-Vietoris sequence to compute the integral cohomology of $M$. We start with

$$
\begin{array}{r}
0 \rightarrow H^{0}(M) \rightarrow H^{0}(U) \oplus H^{0}(V) \rightarrow H^{0}(G) \stackrel{\delta}{\rightarrow} H^{1}(M) \rightarrow H^{1}(U) \oplus H^{1}(V) \rightarrow H^{1}(G) \\
\stackrel{\delta}{\rightarrow} H^{2}(M) \rightarrow H^{2}(U) \oplus H^{2}(V) \rightarrow H^{2}(G) \stackrel{\delta}{\rightarrow} H^{3}(M) \rightarrow 0 .
\end{array}
$$

Clearly the map $H^{0}(U) \oplus H^{0}(V) \rightarrow H^{0}(G)$ is onto. Given the identifications above the map

$$
\varphi: H^{1}(U) \oplus H^{1}(V) \rightarrow H^{1}(G)
$$

becomes

$$
\mathbb{Z} \oplus \mathbb{Z} \ni(n, m) \mapsto n \mu_{1}+m \mu_{2} \in \mathbb{Z}_{G}^{*} .
$$

We therefore have

$0 \rightarrow H^{1}(M) \rightarrow \mathbb{Z} \oplus \mathbb{Z} \stackrel{\varphi}{\rightarrow} \mathbb{Z}_{G}^{*} \stackrel{\delta}{\rightarrow} H^{2}(M) \rightarrow 0 \oplus 0 \rightarrow H^{2}(G) \stackrel{\delta}{\rightarrow} H^{3}(M) \rightarrow 0$ and the lemma follows.

Since $M=\mathbb{R} P^{3}, H^{2}(M, \mathbb{Z})=\mathbb{Z} / 2$. Hence $\mathbb{Z} / 2=\mathbb{Z}_{G}^{*} /\left(\mathbb{Z} \mu_{1}+\mathbb{Z} \mu_{2}\right)$. Consequently, since $\mu_{1}, \mu_{2}$ are primitive, the parallelogram

$$
\left\{a_{1} \mu_{1}+a_{2} \mu_{2} \mid 0 \leq a_{1}, a_{2} \leq 1\right\}
$$

contains exactly five point of $\mathbb{Z}_{G}^{*}$ : four vertices plus the point $\mu=$ $\frac{1}{2}\left(\mu_{1}+\mu_{2}\right)$ in its interior. Hence $\left\{\mu_{1}, \mu\right\}$ is a basis of $\mathbb{Z}_{G}^{*}$. Of course $\mu_{2}=2 \mu-\mu_{1}$.

By the same argument the image $\Psi_{2}(M)$ is an arc in $S^{1}$ of length less than $\pi$ with endpoints $\frac{\nu_{1}}{\left\|\nu_{1}\right\|}, \frac{\nu_{2}}{\left\|\nu_{2}\right\|}$ where $\nu_{1}, \nu_{2} \in \mathbb{Z}_{G}^{*}$ are primitive weights. Moreover $\left\{\nu_{1}, \nu=\frac{1}{2}\left(\nu_{1}+\nu_{2}\right)\right\}$ is a basis of $\mathbb{Z}_{G}^{*}$. The linear map $A: \mathfrak{g}^{*} \rightarrow \mathfrak{g}^{*}$ defined by $A \mu_{1}=\nu_{1}, A \mu=\nu$ is the desired map. 


\section{Bibliography}

[A] C. Albert, Le théorème de réduction de Marsden-Weinstein en géométrie cosymplectique et de contact, J. Geom. Phys. 6 (1989), no. 4, 627-649.

[Ba] A. Banyaga, The geometry surrounding the ArnoldLiouville theorem in Advances in geometry, edited by JeanLuc Brylinski, Ranee Brylinski, Victor Nistor, Boris Tsygan and Ping Xu. Progress in Mathematics, 172. Birkhäuser Boston, Inc., Boston, MA, 1999. xii+399 pp. ISBN 0-81764044-4

[BaM1] A. Banyaga and P. Molino Géométrie des formes de contact complètement intégrables de type toriques in Séminaire Gaston Darboux de Géométrie et Topologie Différentielle, 1991-1992 (Montpellier), 1-25, Univ. Montpellier II, Montpellier, 1993.

[BaM2] A. Banyaga and P. Molino, Complete integrability in contact geometry, Penn State preprint PM 197, 1996.

[Bt] L. Bates, Examples for obstructions to action-angle coordinates, Proc. Roy. Soc. Edinburgh Sect. A 110 (1988), no. $1-2,27-30$.

[BoM] M. Boucetta and P. Molino, Géométrie globale des systèmes hamiltoniens complètement intégrables: fibrations lagrangiennes singulières et coordonnées action-angle à singularités, C. R. Acad. Sci. Paris Sér. I Math. 308 (1989), no. $13,421-424$.

[BG] C. P. Boyer and K. Galicki, A note on toric contact geometry, J. of Geom. and Phys. 35 (2000) 288-298; math.DG/9907043v2.

[D] T. Delzant, Hamiltoniens périodiques et images convexes de l'application moment, Bull. Soc. Math. France 116 (1988), no. $3,315-339$. 
[Ge] H. Geiges, Constructions of contact manifolds, Math. Proc. Cambridge Philos. Soc. 121 (1997), no. 3, 455-464.

[Gi] E. Giroux, Une structure de contact, même tendue, est plus ou moins tordue, Ann. Sci. École Norm. Sup. (4) 27 (1994), no. 6, 697-705.

[GS] V. Guillemin and S. Sternberg, Symplectic techniques in physics, Cambridge University Press, Cambridge - New York, 1984. xi+468 pp. ISBN: 0-521-24866-3

[HS] A. Haefliger and E. Salem, Action of tori on orbifolds, Ann. Global Anal. Geom. 9 (1991), 37-59.

[K] J. L. Koszul, Sur certain groupes de transformations de Lie, dans Colloque de Géométrie Différentielle, Colloque du CNRS 71 (1953), 137-141.

[L1] E. Lerman, A convexity theorem for torus actions on contact manifolds, Ill. J. Math, 46 (2002), 171-184.

http://xxx.lanl.gov/abs/math.SG/0012017.

[L2] E. Lerman, Contact cuts, Israel J. Math, 124 (2001), 7792 ;

http://xxx.lanl.gov/abs/math.SG/0002041.

[L3] E. Lerman, Maximal tori in the contactomorphism groups of circle bundles over Hirzebruch surfaces, Math. Res. Lett., to appear; http://xxx.lanl.gov/abs/math.SG/0204334.

[LS] E. Lerman and N. Shirokova, Toric integrable geodesic flows, Math. Res. Lett. 9 (2002), 105-115; http://xxx.lanl.gov/abs/math.DG/0011139.

[LT] E. Lerman and S. Tolman, Symplectic toric orbifolds, Trans. A.M.S. 349 (1997), 4201-4230.

[Lu] R. Lutz, Sur la géométrie des structures de contact invariantes, Ann. Inst. Fourier (Grenoble), 29 (1979), no. 1, xvii, $283-306$.

[TZ] J. Toth and S. Zelditch, Riemannian manifolds with uniformly bounded eigenfunctions, Duke Math Journal, 111 (2002), 97-132. See http://xxx.lanl.gov/abs/math-ph/0002038.

Received September 18, 2003

Department of Mathematics, University of Illinois, Urbana, IL 61801 E-mail address: lerman@math.uiuc.edu

Partially supported by NSF grant DMS - 980305, the American Institute of Mathematics and R. Kantorovitz. 